\title{
Construction of a Microscopic Model for Yb and Tm Compounds on the Basis of a $j$ - $j$ Coupling Scheme
}

\author{
Takashi HотTA \\ Department of Physics, Tokyo Metropolitan University, 1-1 Minami-Osawa, Hachioji, Tokyo 192-0397, Japan
}

(Received November 6, 2018)

\begin{abstract}
We provide a prescription to construct a microscopic model for heavy lanthanide systems such as $\mathrm{Yb}$ and Tm compounds by exploiting a $j-j$ coupling scheme. Here we consider a situation with a large spinorbit coupling, in which $j=5 / 2$ sextet is fully occupied, while $j=7 / 2$ octet is partially occupied, where $j$ denotes total angular momentum. We evaluate crystalline electric field potentials and Coulomb interactions among the states of the $j=7 / 2$ octet to construct a local Hamiltonian in the $j$ - $j$ coupling scheme. Then, it is found that the local $f$-electron states composed of the $j=7 / 2$ octet agree quite well with those of seven $f$ orbitals even for a realistic value of the spin-orbit coupling. As an example of the application of the present model, we discuss low-temperature multipole states of $\mathrm{Yb}$ - and $\mathrm{Tm}$-based filled skutterudites by analyzing multipole susceptibility of the Anderson model in the $j-j$ coupling scheme with the use of a numerical renormalization group technique. ¿From the comparison with the numerical results of the seven-orbital Anderson model, it is concluded that the multipole state is also well reproduced by the $j$ - $j$ coupling model, even when we include the hybridization between conduction and $f$ electrons for the realistic value of the spin-orbit coupling. Finally, we briefly discuss future applications of the present prescription for theoretical research on heavy lanthanide compounds.
\end{abstract}

KEYWORDS: $j$ - $j$ coupling scheme, microscopic model, heavy electrons, multipole, filled skutterudites

\section{Introduction}

It has been widely recognized that emergence of heavy electron state is understood from the competition in $f$ electron duality nature, ${ }^{1)}$ i.e., itinerancy due to Kondo effect $^{2-4)}$ vs. localization due to Ruderman-Kittel-KasuyaYosida (RKKY) interaction. ${ }^{5-7)}$ There appears a quantum phase transition at zero temperature in the $f$-electron state between itinerant and localized regions. In such a competing region, we have frequently observed unconventional superconductivity and non-Fermi liquid behavior due to the effect of quantum critical fluctuations. ${ }^{8-14)}$ The emphasis on quantum critical nature has been summarized in the famous Doniach's phase diagram, ${ }^{15)}$ which has been a guiding principle for a long time to understand several kinds of anomalous electronic properties of heavy fermion materials.

The concept of quantum critical point seems to be universal, since it holds for $p$-, $d$-, and $f$-electron systems. It is an important issue to accumulate the experimental facts which can be explained by the universal concept of quantum criticality, although it is sometimes difficult to control experimentally quantum criticality in actual materials. However, if we ignore individual characters of electrons such as orbital degree of freedom, in general, difference between $d$ and $f$ electrons cannot be understood only from the control of the ratio of Coulomb interaction and electron bandwidth. It seems to be also important to emphasize individuality of electron in materials, in particular, when we attempt to synthesize new exotic and functional materials.

In this context, we are interested in $\mathrm{Yb}$ and $\mathrm{Tm}$ systems, which have attracted renewed attention due to difference in quantum critical nature between $\mathrm{Ce}$ and $\mathrm{Pr}$ compounds. For trivalent ions, one and two $f$ electrons are included in $\mathrm{Ce}^{3+}$ and $\mathrm{Pr}^{3+}$, respectively, while one and two $f$ holes exist on $\mathrm{Yb}^{3+}$ and $\mathrm{Tm}^{3+}$, respectively. If we use the electron-hole symmetry, we expect similar electronic properties between $f$-electron and hole systems. Such a discussion may be also found in Pr and Tm compounds.

Thus far, unconventional superconductivity has been found in Ce-based materials since the pioneering discovery of superconductivity in $\mathrm{CeCu}_{2} \mathrm{Si}_{2} \cdot{ }^{16)}$ Recently, relatively high superconducting temperature over $2 \mathrm{~K}$ has been also observed in a material group of $\mathrm{CeTIn}_{5}\left(\mathrm{~T}=\mathrm{Ir}, \mathrm{Rh}\right.$, and Co). ${ }^{17-19)}$ Then, if we simply believe the electron-hole picture, at the first glance, it seems to be easy to find superconductivity in $\mathrm{Yb}$ system. However, in spite of much effort to seek for superconductivity in Yb-based heavy-fermion materials, it has been very difficult to synthesize superconducting $\mathrm{Yb}$ compounds. Recently, superconductivity has been observed in $\beta-\mathrm{YbAlB}_{4}$ with a superconducting temperature $T_{\mathrm{c}}=80 \mathrm{mK} .^{20-22)}$ It has been claimed that this material exists just on the quantum critical point at ambient pressure. $\mathrm{In}_{\mathrm{Tm}} \mathrm{Rh}_{6} \mathrm{Sn}_{18}$, superconductivity has been also found with $T_{\mathrm{c}}=2.2 \mathrm{~K} .{ }^{23)}$ Peculiar reentrant properties have been considered to be related to the coexistence of magnetism and superconductivity.

If we emphasize similarity between $\mathrm{Ce}$ and $\mathrm{Yb}$ compounds, we prefer to exploit the concept of quantum criticality on the basis of the electron-hole symmetry. In this case, different quantum critical nature will be explained by the difference in the local interactions and the hybridization between conduction and localized $f$ electrons. However, one may have a simple question whether quantum criticality of one $f$-hole system is really the same as that of one $f$-electron system. In order to clarify $f$-electron state in Yb compounds, we can choose an alternative way to focus on the difference in relevant $f$ electron orbital. This point will be also related to similarity and difference between Pr- and Tm-based compounds.

For the research along such a direction, it is necessary to define the $f$-electron state by a conventional way to include many-body effects. Here we recall a couple of schemes for the description of local $f^{n}$-electron configuration, where $n$ 
denotes the number of $f$ electrons on a localized rare earth or actinide ion. One is an $L S$ coupling scheme, in which we construct the spin $S$ and angular momentum $\boldsymbol{L}$ by following the Hund's rules as $\boldsymbol{S}=\sum_{i=1}^{n} \boldsymbol{s}_{i}$ and $\boldsymbol{L}=\sum_{i=1}^{n} \boldsymbol{\ell}_{i}$, where $\boldsymbol{s}_{i}$ and $\boldsymbol{\ell}_{i}$ are spin and angular momenta for $i$-th $f$ electron, respectively. As is well known, the Hund's rules are based on the Pauli principle and Coulomb interactions among $f$ electrons. After the formation of $\boldsymbol{S}$ and $\boldsymbol{L}$, the effect of spin-orbit interaction is included in the form of $\xi \boldsymbol{L} \cdot \boldsymbol{S}$, where $\xi$ is the spin-orbit coupling in the $L S$ coupling scheme. We note that $\xi>0$ for $n<7$, while $\xi<0$ for $n>7$. Note also that a good quantum number to label such a state is the total angular momentum $\boldsymbol{J}$, which is given by $\boldsymbol{J}=\boldsymbol{L}+\boldsymbol{S}$. Then, the ground state is characterized by $J=|L-S|$ for $n<7$, while $J=L+S$ for $n>7$.

As is understood from the above discussion, the $L S$ coupling scheme is quite useful for the case in which the Hund's rule coupling is much larger than the spin-orbit interaction, since $\boldsymbol{S}$ and $\boldsymbol{L}$ are formed by the Hund's rule coupling prior to the inclusion of the spin-orbit interaction. This assumption is considered to be valid for insulating compounds with localized $f$ electrons. However, when the spin-orbit interaction is not small compared with the Hund's rule coupling, for instance in actinide compounds, the above assumption is not always satisfied. In addition, if the $f$ electrons begin to be itinerant due to hybridization with the conduction electrons, the effect of Coulomb interactions would thereby be effectively reduced. In rough estimation, the effective size of the Coulomb interaction may be as large as the bandwidth of $f$ electrons, leading to a violation of the assumption required for the $L S$ coupling scheme.

For $f$-electron systems in which the spin-orbit interaction becomes larger than the effective Coulomb interactions, we prefer to exploit a $j-j$ coupling scheme. ${ }^{24,25)}$ Here we emphasize that the $j-j$ coupling scheme is convenient for the inclusion of many-body effects by using the standard quantumfield theoretical techniques, since individual $f$-electron states are clearly defined, as we explain below. First, we include the spin-orbit coupling so as to define the state labelled by the total angular momentum $\boldsymbol{j}_{i}$ for the $i$-th $f$ electron, given by $j_{i}=s_{i}+\ell_{i}$. For $f$ orbitals with $\ell=3$, we obtain an octet with $j=7 / 2$ and a sextet with $j=5 / 2$, which are well separated by the spin-orbit interaction. Note that the level for the octet is higher than that of the sextet. Then, we consider the effect of Coulomb interactions to accommodate $n$ electrons among the sextet or octet, leading to the ground state in the $j-j$ coupling scheme. For the models of $\mathrm{Ce}$ and Pr compounds, the sextet should be used for the construction of the effective model. ${ }^{24)}$ On the other hand, for $\mathrm{Yb}$ and Tm materials, since the sextet is fully occupied, we consider the octet to construct the model.

In this paper, we develop a prescription to construct a microscopic effective model for heavy lanthanide systems such as $\mathrm{Yb}$ and $\mathrm{Tm}$ compounds on the basis of the $j-j$ coupling scheme. Then, it is shown that the local $f$-electron state in the $j-j$ coupling scheme agrees quite well with that of the original model including seven $f$ orbitals, even for a realistic value of the spin-orbit coupling. Next we consider the impurity Anderson models to discuss low-temperature multipole states of $f$ electrons. Here we pick up Yb- and Tm-based filled skutterudites as typical examples. The models are analyzed with the use of a numerical renormalization group technique. It is found that the multipole state is well reproduced by the $j-j$ coupling scheme in comparison with that of the sevenorbital Anderson model, even when we include hybridization between conduction and localized $f$ electrons for the realistic value of the spin-orbit coupling.

The organization of this paper is as follows. In Sec. 2, we discuss the local Hamiltonian in the $j-j$ coupling scheme in comparison with the results of the original seven-orbital model. In Sec. 3, we set the impurity Anderson models for filled skutterudites. Then, we show the numerical results for multipole susceptibility to discuss the validity of the $j-j$ coupling model. In Sec. 4, we provide a few comments on future issues on the present prescription and summarize this paper. Throughout this paper, we use such units as $k_{\mathrm{B}}=\hbar=1$.

\section{Local Hamiltonian in a $\boldsymbol{j}$ - $\boldsymbol{j}$ Coupling Scheme}

\subsection{Original Seven-Orbital Model}

In general, the local $f$-electron Hamiltonian is given by

$$
H_{\mathrm{loc}}=H_{\mathrm{so}}+H_{\mathrm{CEF}}+H_{\mathrm{int}} .
$$

The first term denotes the spin-orbit coupling, given by

$$
H_{\mathrm{so}}=\lambda \sum_{m, \sigma, m^{\prime}, \sigma^{\prime}} \zeta_{m, \sigma ; m^{\prime}, \sigma^{\prime}} f_{m \sigma}^{\dagger} f_{m^{\prime} \sigma^{\prime}},
$$

where $\lambda$ is the spin-orbit interaction, $f_{m \sigma}$ is the annihilation operator of $f$ electron, $\sigma=+1(-1)$ for up (down) spin, $m$ is the $z$-component of angular momentum $\ell=3$, and the matrix element $\zeta$ is given by

$$
\begin{aligned}
& \zeta_{m, \sigma ; m, \sigma}=m \sigma / 2, \\
& \zeta_{m+\sigma,-\sigma ; m, \sigma}=\sqrt{\ell(\ell+1)-m(m+\sigma)} / 2,
\end{aligned}
$$

and zero for other cases.

The second term denotes crystalline electric field (CEF) potential, given by

$$
H_{\mathrm{CEF}}=\sum_{m, m^{\prime}, \sigma} B_{m, m^{\prime}} f_{m \sigma}^{\dagger} f_{m^{\prime} \sigma},
$$

where $B_{m, m^{\prime}}$ is determined from the CEF table for $J=\ell=3 .^{26,27)}$ Note that electrostatic CEF potentials do not act on $f$-electron spin. Since we will consider later the multipole state of heavy lanthanide filled skutterudites, here we show $B_{m, m^{\prime}}$ of the cubic system with $T_{\mathrm{h}}$ symmetry. ${ }^{28)}$ The results are given by

$$
\begin{aligned}
& B_{3,3}=B_{-3,-3}=180 B_{4}^{0}+180 B_{6}^{0}, \\
& B_{2,2}=B_{-2,-2}=-420 B_{4}^{0}-1080 B_{6}^{0}, \\
& B_{1,1}=B_{-1,-1}=60 B_{4}^{0}+2700 B_{6}^{0}, \\
& B_{0,0}=360 B_{4}^{0}-3600 B_{6}^{0}, \\
& B_{3,-1}=B_{-3,1}=12 \sqrt{15}\left(B_{4}^{4}+5 B_{6}^{4}\right), \\
& B_{2,-2}=60 B_{4}^{4}-360 B_{6}^{4}, \\
& B_{3,1}=B_{-3,-1}=24 \sqrt{15} B_{6}^{2}, \\
& B_{2,0}=B_{-2,0}=-48 \sqrt{30} B_{6}^{2}, \\
& B_{1,-1}=360 B_{6}^{2}, \\
& B_{3,-3}=360 B_{6}^{6},
\end{aligned}
$$

Note the relation of $B_{m, m^{\prime}}=B_{m^{\prime}, m}$. We also note the relations of $B_{4}^{4}=5 B_{4}^{0}, B_{6}^{4}=-21 B_{6}^{0}$, and $B_{6}^{6}=-B_{6}^{2}$. Following the traditional notation, ${ }^{29)}$ we define

$$
\begin{aligned}
& B_{4}^{0}=W x / F(4), \\
& B_{6}^{0}=W(1-|x|) / F(6), \\
& B_{6}^{2}=W y / F^{t}(6),
\end{aligned}
$$

where $x, y$, and the sign of $W$ specify the CEF scheme 
for $T_{\mathrm{h}}$ point group, ${ }^{28)}$ while the absolute value of $W$ determines the energy scale of the CEF potential. Concerning nondimensional parameters, $F(4)$ and $F(6)$, we choose $F(4)=15$, $F(6)=180$, and $F^{t}(6)=24$ for $J=3$.

Finally, $H_{\text {int }}$ denotes Coulomb interaction term, given by

$$
H_{\mathrm{int}}=\sum_{m_{1} \sim m_{4} \sigma, \sigma^{\prime}} \sum_{m_{1} m_{2}, m_{3} m_{4}} f_{m_{1} \sigma}^{\dagger} f_{m_{2} \sigma^{\prime}}^{\dagger} f_{m_{3} \sigma^{\prime}} f_{m_{4} \sigma},
$$

where the Coulomb integral $I_{m_{1} m_{2}, m_{3} m_{4}}$ is expressed by

$$
I_{m_{1} m_{2}, m_{3} m_{4}}=\sum_{k=0}^{6} F^{k} c_{k}\left(m_{1}, m_{4}\right) c_{k}\left(m_{2}, m_{3}\right) .
$$

Here $F^{k}$ is the Slater-Condon parameter ${ }^{30,31)}$ and $c_{k}$ is the Gaunt coefficient ${ }^{32,33)}$ which is tabulated in the standard textbooks of quantum mechanics. ${ }^{34)}$ Note that the sum is limited by the Wigner-Eckart theorem to $k=0,2,4$, and 6 .

\subsection{Effective Hamiltonian for $j=7 / 2$ Octet}

In order to obtain the model in the $j-j$ coupling scheme, we transform the $f$-electron basis between $(m, \sigma)$ and $(j, \mu)$ representations, connected by Clebsch-Gordan coefficients, where $j$ is the total angular momentum and $\mu$ is the $z$ component of $j$. When we define $f_{j \mu}$ as the annihilation operator for $f$ electron labelled by $j$ and $\mu$, the transformation is given by

$$
f_{j \mu}=\sum_{m, \sigma} C_{j, \mu ; m, \sigma} f_{m \sigma},
$$

where the Clebsch-Gordan coefficient $C_{j, \mu ; m, \sigma}$ is give by

$$
\begin{aligned}
& C_{5 / 2, \mu ; \mu-\sigma / 2, \sigma}=-\sigma \sqrt{(7 / 2-\sigma \mu) / 7}, \\
& C_{7 / 2, \mu ; \mu-\sigma / 2, \sigma}=\sqrt{(7 / 2+\sigma \mu) / 7},
\end{aligned}
$$

and other components are zero.

After the transformation, the spin-orbit coupling term is diagonalized as

$$
\tilde{H}_{\text {so }}=\sum_{j, \mu} \tilde{\lambda}_{j} f_{j \mu}^{\dagger} f_{j \mu}
$$

with $\tilde{\lambda}_{5 / 2}=-2 \lambda$ and $\tilde{\lambda}_{7 / 2}=(3 / 2) \lambda$. The CEF and Coulomb interaction terms are, respectively, given by

$$
\tilde{H}_{\mathrm{CEF}}=\sum_{j_{1} \mu_{1}, j_{2} \mu_{2}} \tilde{B}_{\mu_{1}, \mu_{2}}^{j_{1}, j_{2}} f_{j_{1} \mu_{1}}^{\dagger} f_{j_{2} \mu_{2}},
$$

and

$$
\tilde{H}_{\text {int }}=\sum_{j_{1} \sim j_{4}} \sum_{\mu_{1} \sim \mu_{4}} \tilde{I}_{\mu_{1}, \mu_{2} ; \mu_{3}, \mu_{4}}^{j_{1}, j_{2} ; j_{3}, j_{4}} f_{j_{1} \mu_{1}}^{\dagger} f_{j_{2} \mu_{2}}^{\dagger} f_{j_{3} \mu_{3}} f_{j_{4} \mu_{4}},
$$

where $\tilde{B}$ and $\tilde{I}$ are the CEF potential and Coulomb interaction, respectively, in the basis of $j$ and $\mu$.

In the present paper, we consider the model for heavy lanthanide systems with $n>7$. In the limit of large $\lambda$ for the $j-j$ coupling scheme, $j=5 / 2$ sextet is fully occupied, while $j=7 / 2$ octet is partially occupied. Thus, here we simply discard all the $j=5 / 2$ states and keep only the $j=7 / 2$ octet. Namely, we accommodate $n-6$ electrons in the $j=7 / 2$ octet. Note that in this approximation, the spin-orbit coupling is given by the effect of potential energy which does not depend on the orbitals. Since such an energy can be included in the chemical potential shift, we do not consider explicitly $\tilde{H}_{\text {so }}$ in the following. Hereafter, we suppress the subscription $j$ in $f_{j \mu}$, since we con- sider only the $j=7 / 2$ octet.

The local model in the $j-j$ coupling scheme is given by

$$
\tilde{H}_{\mathrm{loc}}=\tilde{H}_{\mathrm{CEF}}+\tilde{H}_{\mathrm{int}},
$$

where $\tilde{H}_{\mathrm{CEF}}$ is the CEF potential in the $j=7 / 2$ octet, given as

$$
\tilde{H}_{\mathrm{CEF}}=\sum_{\mu, \nu} \tilde{B}_{\mu, \nu} f_{\mu}^{\dagger} f_{\nu}
$$

Here $f_{\mu}^{\dagger}$ is the creation operator of $f$ electron in the $\mu$-state and $\mu$ indicates the $z$-component of the total angular momentum which specifies the state in the $j=7 / 2$ octet. The CEF potential in the $j=7 / 2$ octet is given by ${ }^{27)}$

$$
\begin{aligned}
& \tilde{B}_{7 / 2,7 / 2}=\tilde{B}_{-7 / 2,-7 / 2}=420 \tilde{B}_{4}^{0}+1260 \tilde{B}_{6}^{0}, \\
& \tilde{B}_{5 / 2,5 / 2}=\tilde{B}_{-5 / 2,-5 / 2}=-780 \tilde{B}_{4}^{0}-6300 \tilde{B}_{6}^{0}, \\
& \tilde{B}_{3 / 2,3 / 2}=\tilde{B}_{-3 / 2,-3 / 2}=-180 \tilde{B}_{4}^{0}+11340 \tilde{B}_{6}^{0}, \\
& \tilde{B}_{1 / 2,1 / 2}=\tilde{B}_{-1 / 2,-1 / 2}=540 \tilde{B}_{4}^{0}-6300 \tilde{B}_{6}^{0}, \\
& \tilde{B}_{7 / 2,-1 / 2}=B_{-7 / 2,1 / 2}=12 \sqrt{35}\left(\tilde{B}_{4}^{4}+15 \tilde{B}_{6}^{4}\right), \\
& \tilde{B}_{5 / 2,-3 / 2}=B_{-5 / 2,3 / 2}=60 \sqrt{3}\left(\tilde{B}_{4}^{4}-7 \tilde{B}_{6}^{4}\right), \\
& \tilde{B}_{7 / 2,3 / 2}=B_{-7 / 2,-3 / 2}=120 \sqrt{21} \tilde{B}_{6}^{2}, \\
& \tilde{B}_{5 / 2,1 / 2}=B_{-5 / 2,-1 / 2}=-504 \sqrt{5} \tilde{B}_{6}^{2}, \\
& \tilde{B}_{3 / 2,-1 / 2}=B_{-3 / 2,1 / 2}=168 \sqrt{15} \tilde{B}_{6}^{2}, \\
& \tilde{B}_{7 / 2,-5 / 2}=B_{-7 / 2,5 / 2}=360 \sqrt{7} \tilde{B}_{6}^{6},
\end{aligned}
$$

where we note again the relations of $\tilde{B}_{4}^{4}=5 \tilde{B}_{4}^{0}, \tilde{B}_{6}^{4}=-21 \tilde{B}_{6}^{0}$, and $\tilde{B}_{6}^{6}=-\tilde{B}_{6}^{2}$. The CEF parameters for $j=7 / 2$ are related to those for $J=\ell=3$ as

$$
\begin{aligned}
& \tilde{B}_{4}^{0}=\left(\beta_{7 / 2} / \beta_{3}\right) B_{4}^{0}=3 B_{4}^{0} / 7, \\
& \tilde{B}_{6}^{0}=\left(\gamma_{7 / 2} / \gamma_{3}\right) B_{6}^{0}=B_{6}^{0} / 7, \\
& \tilde{B}_{6}^{2}=\left(\gamma_{7 / 2} / \gamma_{3}\right) B_{6}^{2}=B_{6}^{2} / 7,
\end{aligned}
$$

where $\beta_{J}$ and $\gamma_{J}$ are fourth- and sixth-order Stevens factors, respectively. ${ }^{26)}$ We note that $\beta_{3}=2 / 495, \beta_{7 / 2}=2 / 1155$, $\gamma_{3}=-4 / 3861$, and $\gamma_{7 / 2}=-4 / 27027$.

The second term in eq. (14) indicates the Coulomb interactions in the $j=7 / 2$ octet, which is given by

$$
\tilde{H}_{\mathrm{int}}=\sum_{\mu, \nu, \mu^{\prime}, \nu^{\prime}} \tilde{I}_{\mu, \nu ; \nu^{\prime}, \mu^{\prime}} f_{\mu}^{\dagger} f_{\nu}^{\dagger} f_{\nu^{\prime}} f_{\mu^{\prime}}
$$

where $\tilde{I}$ is the matrix element for Coulomb interactions among $j=7 / 2$ states. In order to classify the Coulomb interactions in the $j=7 / 2$ octet, we consider the situation where we accommodate two electrons in the octet. Note that the allowed values for total angular momentum $J$ are $0,2,4$, and 6 due to the Pauli principle. Thus, the Coulomb interaction term should be written in a $28 \times 28$ matrix form. Note that " 28 " is the sum of the basis numbers for singlet $(J=0)$, quintet $(J=2)$, nonet $(J=4)$, and tridectet $(J=6)$. As is easily understood, this $28 \times 28$ matrix can be decomposed into a blockdiagonalized form labelled by $J_{z}$, including one $4 \times 4$ matrix for $J_{z}=0$, four $3 \times 3$ matrices for $J_{z}= \pm 1$ and \pm 2 , four $2 \times 2$ matrices for $J_{z}= \pm 3$ and \pm 4 , and four $1 \times 1$ for $J_{z}= \pm 5$ and \pm 6 . We skip the details of tedious calculations for the evaluation of matrix elements and show only the results by using the parameters $E_{k}(k=0,1,2,3),{ }^{35,36)}$ which are related to the Slater-Condon parameters $F^{k}$ as ${ }^{37)}$

$$
\begin{aligned}
& E_{0}=F^{0}-\frac{25}{567} F^{2}-\frac{5}{231} F^{4}-\frac{125}{11583} F^{6}, \\
& E_{1}=\frac{40}{567} F^{2}+\frac{8}{231} F^{4}+\frac{200}{11583} F^{6}, \\
& E_{2}=\frac{2}{1617} F^{2}-\frac{2}{5929} F^{4}, \\
& E_{3}=\frac{10}{43659} F^{2}+\frac{2}{17787} F^{4}-\frac{100}{1656369} F^{6} .
\end{aligned}
$$


For $J_{z}=6$ and 5 , we obtain

$$
\tilde{I}_{7 / 2,5 / 2 ; 5 / 2,7 / 2}=E_{0}-154 E_{3},
$$

and

$$
\tilde{I}_{7 / 2,3 / 2 ; 3 / 2,7 / 2}=E_{0}-154 E_{3},
$$

respectively. For $J_{z}=4$ and 3, we obtain

$$
\begin{aligned}
& \tilde{I}_{7 / 2,1 / 2 ; 1 / 2,7 / 2}=E_{0}-35 E_{2} / 2-119 E_{3} / 2, \\
& \tilde{I}_{5 / 2,3 / 2 ; 3 / 2,5 / 2}=E_{0}-75 E_{2} / 2+97 E_{3} / 2, \\
& \tilde{I}_{7 / 2,1 / 2 ; 3 / 2,5 / 2}=\sqrt{105}\left(5 E_{2}-27 E_{3}\right) / 2,
\end{aligned}
$$

and

$$
\begin{aligned}
& \tilde{I}_{7 / 2,-1 / 2 ;-1 / 2,7 / 2}=E_{0}-35 E_{2}+35 E_{3}, \\
& \tilde{I}_{5 / 2,1 / 2 ; 1 / 2,5 / 2}=E_{0}-20 E_{2}-46 E_{3}, \\
& \tilde{I}_{7 / 2,-1 / 2 ; 1 / 2,5 / 2}=\sqrt{7}\left(10 E_{2}-54 E_{3}\right) .
\end{aligned}
$$

For $J_{z}=2$ and 1 , we obtain

$$
\begin{aligned}
& \tilde{I}_{7 / 2,-3 / 2 ;-3 / 2,7 / 2}=E_{0}-21 E_{2}+98 E_{3}, \\
& \tilde{I}_{5 / 2,-1 / 2 ;-1 / 2,5 / 2}=E_{0}+35 E_{2}-46 E_{3}, \\
& \tilde{I}_{3 / 2,1 / 2 ; 1 / 2,3 / 2}=E_{0}+30 E_{2}+80 E_{3}, \\
& \tilde{I}_{7 / 2,-3 / 2 ;-1 / 2,5 / 2}=-\sqrt{105}\left(2 E_{2}+9 E_{3}\right), \\
& \tilde{I}_{7 / 2,-3 / 2 ; 1 / 2,3 / 2}=9 \sqrt{35}\left(E_{2}-E_{3}\right), \\
& \tilde{I}_{5 / 2,-1 / 2 ; 1 / 2,3 / 2}=-\sqrt{3}\left(25 E_{2}+63 E_{3}\right),
\end{aligned}
$$

and

$$
\begin{aligned}
& \tilde{I}_{7 / 2,-5 / 2 ;-5 / 2,7 / 2}=E_{0}+49 E_{2} / 2+259 E_{3} / 2, \\
& \tilde{I}_{5 / 2,-3 / 2 ;-3 / 2,5 / 2}=E_{0}+27 E_{2}+17 E_{3}, \\
& \tilde{I}_{3 / 2,-1 / 2 ;-1 / 2,3 / 2}=E_{0}-15 E_{2} / 2-29 E_{3} / 2, \\
& \tilde{I}_{7 / 2,-5 / 2 ;-3 / 2,5 / 2}=-\sqrt{21}\left(13 E_{2}+9 E_{3}\right), \\
& \tilde{I}_{7 / 2,-5 / 2 ;-1 / 2,3 / 2}=9 \sqrt{105}\left(E_{2}-E_{3}\right) / 2, \\
& \tilde{I}_{5 / 2,-3 / 2 ;-1 / 2,3 / 2}=-3 \sqrt{5}\left(E_{2}+21 E_{3}\right),
\end{aligned}
$$

Finally, for $J_{z}=0$, we obtain

$$
\begin{aligned}
& \tilde{I}_{7 / 2,-7 / 2 ;-7 / 2,7 / 2}=E_{0}+E_{1}+49 E_{2}+105 E_{3}, \\
& \tilde{I}_{3 / 2,-3 / 2 ;-3 / 2,3 / 2}=E_{0}+E_{1}-29 E_{2}+51 E_{3}, \\
& \tilde{I}_{5 / 2,-5 / 2 ;-5 / 2,5 / 2}=E_{0}+E_{1}+9 E_{2}-75 E_{3}, \\
& \tilde{I}_{1 / 2,-1 / 2 ;-1 / 2,1 / 2}=E_{0}+E_{1}+15 E_{2}+51 E_{3}, \\
& \tilde{I}_{7 / 2,-7 / 2 ;-5 / 2,5 / 2}=-E_{1}-49 E_{2} / 2+49 E_{3} / 2, \\
& \tilde{I}_{7 / 2,-7 / 2 ;-3 / 2,3 / 2}=E_{1}-21 E_{2}-56 E_{3}, \\
& \tilde{I}_{7 / 2,-7 / 2 ;-1 / 2,1 / 2}=-E_{1}+105 E_{2} / 2+49 E_{3} / 2, \\
& \tilde{I}_{5 / 2,-5 / 2 ;-3 / 2,3 / 2}=-E_{1}+21 E_{2} / 2-131 E_{3} / 2, \\
& \tilde{I}_{5 / 2,-5 / 2 ;-1 / 2,1 / 2}=E_{1}+15 E_{2} / 2-92 E_{3}, \\
& \tilde{I}_{3 / 2,-3 / 2 ;-1 / 2,1 / 2}=-E_{1}-45 E_{2} / 2-131 E_{3} / 2,
\end{aligned}
$$

Note here the following relations:

$$
\tilde{I}_{\mu, \nu ; \nu^{\prime}, \mu^{\prime}}=\tilde{I}_{\mu^{\prime}, \nu^{\prime} ; \nu, \mu},
$$

and

$$
\tilde{I}_{\mu, \nu ; \nu^{\prime}, \mu^{\prime}}=\tilde{I}_{-\nu,-\mu ;-\mu^{\prime},-\nu^{\prime}} .
$$

By using these two relations and eqs. (20)-(26), we can obtain all the Coulomb matrix elements.

\subsection{CEF Energy Levels}

Let us now consider the situation in which two electrons are accommodated in the $j=7 / 2$ octet. This situation indicates the case with 8 electrons in $f$ orbitals, corresponding to $f^{8}$ configuration of $\mathrm{Tb}^{3+}$ ion. When we diagonalize the $28 \times 28$ matrix for Coulomb interaction terms, we can easily obtain the eigen energies as $E_{0}-154 E_{3}$ for the $J=6$ tridectet,
$E_{0}-55 E_{2}+143 E_{3}$ for the $J=4$ nonet, $E_{0}+99 E_{2}+143 E_{3}$ for the $J=2$ quintet, and $E_{0}+4 E_{1}$ for the $J=0$ singlet. These values are exactly the same as those obtained in the nuclear shell theory in the $j-j$ coupling scheme. ${ }^{38)}$ For typical values of Slater-Condon parameters, we find that the ground state is specified by $J=6$ in the $j-j$ coupling scheme. For $\mathrm{Tb}^{3+}$ ion, in the $L S$ coupling scheme, we obtain the ground-state level as ${ }^{7} F$ with $S=3$ and $L=3$ from the Hund's rules. On further inclusion of the spin-orbit interaction, the ground state becomes characterized by $J=6$, expressed as ${ }^{7} F_{6}$ in the traditional notation. Note that we are considering a two-electron problem. Thus, when we correctly include the effects of Coulomb interactions, the same quantum number as that in the $L S$ coupling scheme is obtained in the $j-j$ coupling scheme for the groundstate multiplet.

In order to discuss the CEF energy levels, it is necessary to determine the values of local interactions. Among them, concerning the Slater-Condon parameters, we set $F^{0}=10 \mathrm{eV}$ by hand. The magnitude of $F^{0}$ is related to the absolute value of the ground state energy. It can be evaluated by the firstprinciples calculation, but it is out of the scope of the present paper. Other Slater-Condon parameters are determined so as to reproduce excitation spectra of $\operatorname{Pr}^{3+}$ ion. ${ }^{39,40)}$ After the fitting, we obtain $F^{2}=8.75 \mathrm{eV}, F^{4}=6.60 \mathrm{eV}$, and $F^{6}=4.44$ $\mathrm{eV}^{41,42)}$ As long as we ignore the difference in lanthanide ions, e.g., the size of ion radius, we use these values for all lanthanide ions. On the other hand, as for the spin-orbit coupling $\lambda$, we use the value which has been determined experimentally for each lanthanide ion. ${ }^{43)}$

Concerning CEF parameters, it is necessary to specify the actual material, since they depend on the crystal structure and the kinds of ligand ions. Here we consider the case of filled skutterudite materials, since we will analyze the multipole state of filled skutterudites later in this paper. The CEF parameters are different from material to material even if we fix the material group, but the typical values are $W=-0.4 \mathrm{meV}$, $y=0.3$, and $x=0.3$, which are determined so as to reproduce quasi-quartet $\mathrm{CEF}$ scheme of $\mathrm{PrOs}_{4} \mathrm{Sb}_{12} \cdot{ }^{44-46)}$ Note that for Pr atom, we use $\lambda=0.095 \mathrm{eV}$ from the experimental value.

In Fig. 1(a), we show the results of CEF energy levels vs. $x$ for the case of $n=2$, corresponding to $\operatorname{Pr}^{3+}$, with the use of the above parameters. The vertical dash line denotes the position of $x=0.3$ and we can understand that the ground state is $\Gamma_{1}^{+}$singlet and the first excited state is $\Gamma_{4}^{+(2)}$ triplet with the small excitation energy. This is considered to be a typical situation of $\mathrm{PrOs}_{4} \mathrm{Sb}_{12}$. When we change the values of $x$ and/or $y$, we can obtain another situation for different filled skutterudite material.

In Fig. 1(b), we show the CEF energy levels vs. $x$ for the case of $n=13$ with $W=-0.4 \mathrm{meV}$ and $y=0.3$. The spin-orbit coupling $\lambda$ is set as $0.356 \mathrm{eV}$ for $\mathrm{Yb}$ atom. ${ }^{43)}$ In the $O_{\mathrm{h}}$ point group, the octet is known to split into two doublets and one quartet. ${ }^{29)}$ In the $T_{\mathrm{h}}$ point group, on the other hand, two doublets are mixed. ${ }^{28)}$ In the present parameters, we always find the doublet ground state, irrespective of the values of $x$.

In Figs. 2, we show the results for the case of $n=12$ corresponding to $\mathrm{Tm}^{3+}$ ion, in order to see the validity of the $j-j$ coupling scheme. In Fig. 2(a), the result for the original seven-orbital model in eq. (1) is shown. In the $O_{\mathrm{h}}$ group, the tridectet of $J=6$ is split into two singlets, one doublet, and 

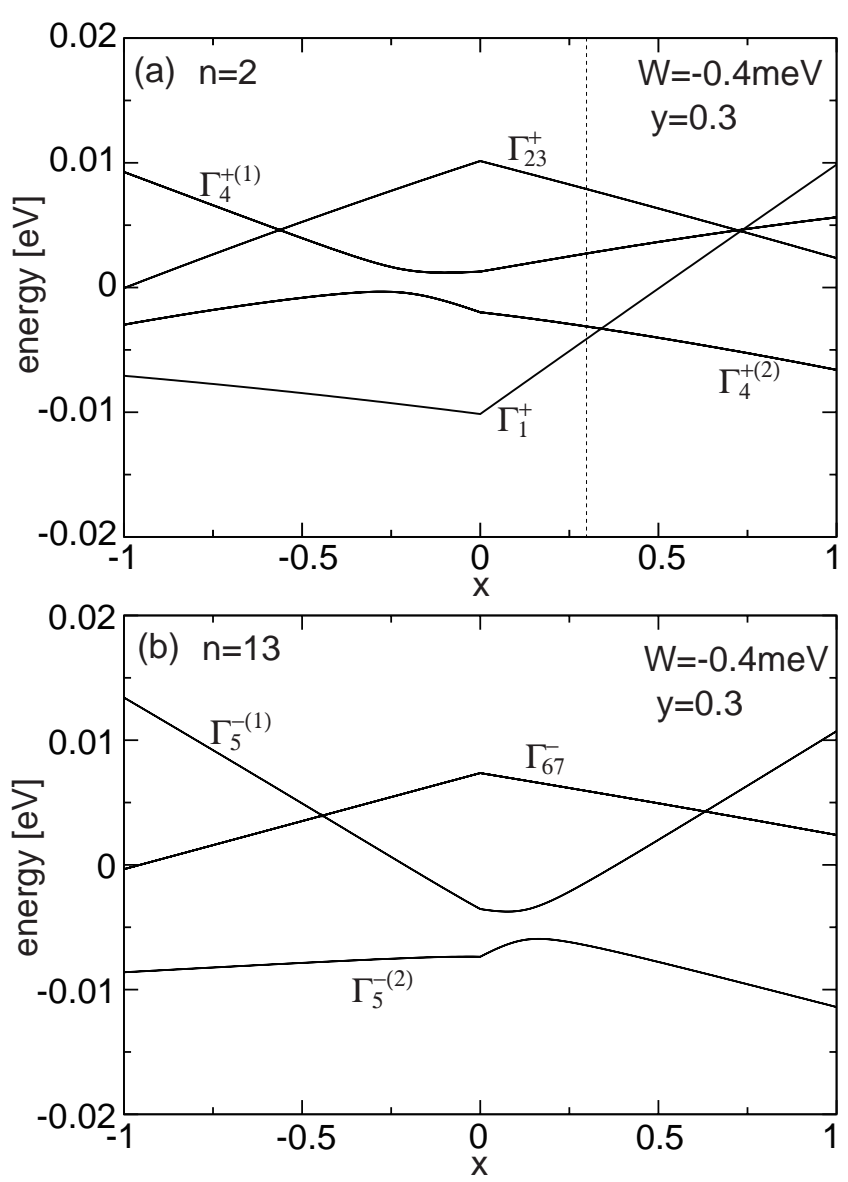

Fig. 1. CEF energy levels for (a) $n=2$ and (b) $n=13$. The vertical line denote the position of $x=0.3$. Concerning other parameters, see the main text.

three triplets. ${ }^{29)}$ In the $T_{\mathrm{h}}$ group, on the other hand, two singlets are mixed. Three triplets are also mixed due to the $y$ term. In the present parameters, the ground state around at $x=0.3$ is characterized by $\Gamma_{1}^{+}$singlet. However, when we increase the value of $x$, we find the change of the ground state from $\Gamma_{1}^{+}$singlet to $\Gamma_{23}^{+}$non-Kramers doublets. Such a change is found to occur around at $x \sim 0.9$.

In Fig. 2(b), we show the CEF energies vs. $x$ of the $j-j$ coupling model eq. (14) with the use of the same parameters, except for the value of the spin-orbit coupling. In the $j-j$ coupling scheme, $\lambda$ is set as infinity. Nevertheless, in the first impression, even if the actual value of $\lambda$ is finite, the results of the $j$ - $j$ coupling model agree quite well with those of the original seven-orbital model in Fig. 2(a). If we effectively change the absolute value of $W$ as a fitting parameter, Fig. 2(a) can be reproduced by the $j-j$ coupling model quantitatively.

The reason why the CEF energy levels are well reproduced by the $j-j$ coupling scheme even for the finite value of $\lambda$ is as follows. Since the maximum value of the difference in the $z$-component of total angular momentum is seven among $j=7 / 2$ states, the sixth-order CEF potential can be included in the $j-j$ coupling scheme, in sharp contrast to the case of $j=5 / 2$. $^{24)}$ Thus, the difference in the value of $\lambda$ does not provide serious effect on the CEF ground state, as long as we consider $\lambda \gg|W|$. In fact, after lengthy algebraic calculations, we obtain the overlap integral of the ground states for $n=12$ as $\left\langle\Phi \mid \Phi_{j-j}\right\rangle=\sqrt{6 / 7}=0.926,{ }^{35,36)}$ where $|\Phi\rangle$ and $\left|\Phi_{j-j}\right\rangle$ de-
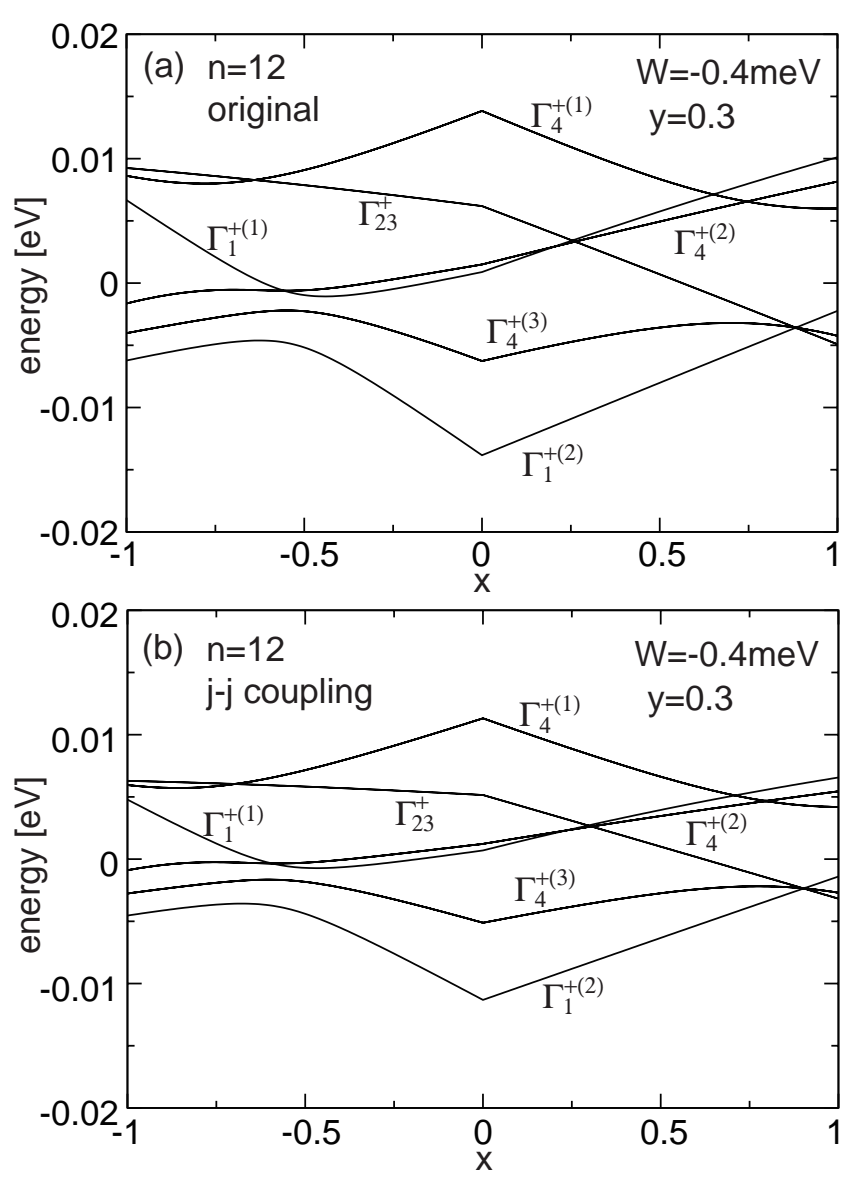

Fig. 2. CEF energy levels vs. $x$ for $n=12$ (a) in the original seven-orbital model eq. (1) and (b) in the $j-j$ coupling model eq. (14).

note the CEF ground state of the seven-orbital model and the $j-j$ coupling one, respectively.

We do not show further the results for the cases of $n<12$, but the CEF states of the $j$ - $j$ coupling model can reproduce well those of the original seven-orbital model. Thus, we conclude that the local $f$-electron state of heavy lanthanide systems is well approximated by the $j-j$ coupling scheme. This is one of important messages of the present paper.

\section{Numerical Analysis of Impurity Anderson Models}

We have explained the prescription to obtain the local Hamiltonian in the $j-j$ coupling scheme. Even if we use only the $j=7 / 2$ octet, it is possible to reproduce local multi- $f$ electron state, which agrees well with those obtained in the original seven-orbital model. By including further the itinerancy of $f$ electrons, we can discuss magnetism and superconductivity of heavy lanthanide compounds from a microscopic viewpoint. In this section, as an example, we discuss the multipole state of $\mathrm{Yb}$ - and Tm-based filled skutterudites by using the Anderson models. Then, we show the effectiveness of the $j-j$ coupling model in the microscopic level.

\subsection{Anderson Models}

The seven-orbital Anderson Hamiltonian is given by

$H=\sum_{\boldsymbol{k}, \sigma} \varepsilon_{\boldsymbol{k}} c_{\boldsymbol{k} \sigma}^{\dagger} c_{\boldsymbol{k} \sigma}+\sum_{\boldsymbol{k}, \sigma, m}\left(V_{m} c_{\boldsymbol{k} \sigma}^{\dagger} f_{m \sigma}+\right.$ h.c. $)+H_{\mathrm{loc}}$ 
where $\varepsilon_{\boldsymbol{k}}$ denotes conduction electron dispersion, $c_{\boldsymbol{k} \sigma}$ indicates the annihilation operator for conduction electron with momentum $\boldsymbol{k}$ and spin $\sigma, V_{m}$ is the hybridization between conduction and $f$ electrons, and the local $f$-electron term $H_{\text {loc }}$ is already given in eq. (1). For filled skutterudites, the main conduction band is given by $a_{\mathrm{u}}$, constructed from $p$-orbitals of pnictogen. ${ }^{47}$ Note that the hybridization occurs between the states with the same symmetry. Since the $a_{\mathrm{u}}$ conduction band has xyz symmetry, we set $V_{2}=V / \sqrt{2}$, $V_{-2}=-V / \sqrt{2}$, and zeros for other values of $m$. The hybridization is fixed as $V=0.05 \mathrm{eV}$ and a half of the bandwidth of $a_{\mathrm{u}}$ conduction band is set as $1 \mathrm{eV}$.

The $j$ - $j$ coupling Anderson model is given by

$$
\tilde{H}=\sum_{\boldsymbol{k}, \sigma} \varepsilon_{\boldsymbol{k}} c_{\boldsymbol{k} \sigma}^{\dagger} c_{\boldsymbol{k} \sigma}+\sum_{\boldsymbol{k}, \sigma, \mu}\left(\tilde{V}_{\sigma, \mu} c_{\boldsymbol{k} \sigma}^{\dagger} f_{\mu}+\text { h.c. }\right)+\tilde{H}_{\mathrm{loc}},
$$

where the local $f$-electron term in the $j-j$ coupling scheme $\tilde{H}_{\text {loc }}$ is given by eq. (14). Since the $a_{\mathrm{u}}$ conduction band has xyz symmetry, which is described by $\Gamma_{5}^{-}$in the $T_{\mathrm{h}}$ group, i.e., $\Gamma_{7}^{-}$in the $O_{\mathrm{h}}$ group, we set $\tilde{V}_{\uparrow, 5 / 2}=V \sqrt{3} / 2, \tilde{V}_{\uparrow,-3 / 2}=-V / 2$, $\tilde{V}_{\downarrow,-5 / 2}=-V \sqrt{3} / 2, \tilde{V}_{\downarrow, 3 / 2}=V / 2$, and zeros for other cases. Note that the connectivity between $\mu$ and $\sigma$ is determined by the definition of pseudo-spin of $f$-electron state on the basis of the time reversal symmetry. For the $j-j$ coupling model, we also set $V=0.05 \mathrm{eV}$ for simplicity, since this value is not so important at low enough temperatures in the following discussion. Note that for the $T_{\mathrm{h}}$ group for filled skutterudites, $\Gamma_{6}^{-}$and $\Gamma_{7}^{-}$doublets in the $O_{\mathrm{h}}$ group are mixed and they are expressed as $\Gamma_{5}^{-(1)}$ and $\Gamma_{5}^{-(2)}$.

It should be noted that we do not show explicitly the chemical potential terms both in the models, but in actual calculations, we set the value of the chemical potential so as to fix the local $f$-electron number as $n=13$ or 12 .

\subsection{Multipole Operator}

In order to discuss the multipole state, it is necessary to define the multipole operator. The details can be found in Refs. 41 and 42, but here we briefly explain the method to define the multipole operator to make this paper self-contained with some additional comments.

When we consider multipole operator for $f$ electrons, it should be defined in the one-body form as an extension of charge and total angular momentum operators on the basis of a belief that the multipole denotes the combined degree of freedom of spin and orbital. ${ }^{42)}$

In the multipole expansion of potential in electromagnetism, higher electric and magnetic multipole moments appear in the coefficients of the expansion by the spherical harmonics $Y_{L M}$ with larger angular momentum. In group theory, $Y_{L M}$ is defined by the basis of irreducible representation $D^{(L)}$ of the rotation group $R$, expressed as

$$
R Y_{L M}=\sum_{M^{\prime}} Y_{L M^{\prime}} D_{M M^{\prime}}^{(L)}
$$

In order to define $f$-electron multipole operator, on the analogy of the multipole expansion, we exploit a concept of spherical tensor operator in the quantum mechanics of angular momentum. ${ }^{48)}$ When we consider the rotation of operator $\hat{T}$, we obtain a set of operators $\hat{T}^{(k)}=\left\{\hat{T}_{q}^{(k)}\right\}$ with $(2 k+1)$ - components $(q=-k,-k+1, \cdots, k-1, k)$, given by

$$
R \hat{T}_{q}^{(k)} R^{-1}=\sum_{q^{\prime}} \hat{T}_{q^{\prime}}^{(k)} D_{q q^{\prime}}^{(k)}
$$

Namely, $\hat{T}_{q}^{(k)}$ is transformed like a basis of irreducible representation $D^{(k)}$ for the rotation. Such $\hat{T}_{q}^{(k)}$ is called spherical tensor operator of rank $k$.

Thus far, we have implicitly assumed $f$-electron density in an isolated ion, but in actuality, rare-earth ions are put in the crystal structure. Then, it is convenient to change from spherical to cubic tensor operators, given by

$$
\hat{T}_{\gamma}^{(k)}=\sum_{q} G_{\gamma, q}^{(k)} \hat{T}_{q}^{(k)}
$$

where $k$ is a rank of multipole, an integer $q$ runs between $-k$ and $k, \gamma$ is a label to express $O_{\mathrm{h}}$ irreducible representation, and $G_{\gamma, q}^{(k)}$ is the transformation matrix between spherical and cubic harmonics. Then, the cubic tensor operator for $f$ electron is expressed in the second-quantized form as

$$
\hat{T}_{\gamma}^{(k)}=\sum_{m \sigma, m^{\prime} \sigma^{\prime}} T_{m \sigma, m^{\prime} \sigma^{\prime}}^{(k, \gamma)} f_{m \sigma}^{\dagger} f_{m^{\prime} \sigma^{\prime}}
$$

Throughout this paper, we use the cubic tensor operator as multipole.

As for the classification of multipole, we use the notations in the group theory. We express the irreducible representation of the CEF state by Bethe notation in this paper, but for multipoles, we use short-hand notations by the combination of the number of irreducible representation and the parity of time reversal symmetry, $\mathrm{g}$ for gerade and $\mathrm{u}$ for ungerade. Note also that for the $T_{\mathrm{h}}$ group, $\Gamma_{1}$ and $\Gamma_{2}$ of $O_{\mathrm{h}}$ are mixed. We remark that $\Gamma_{4}$ and $\Gamma_{5}$ of $O_{\mathrm{h}}$ are also mixed in $T_{\mathrm{h}}$. Thus, we obtain six independent multipole components as $1 \mathrm{~g}+2 \mathrm{~g}, 2 \mathrm{u}, 3 \mathrm{~g}, 3 \mathrm{u}$, $4 g+5 g$, and $4 u+5 u$ for filled skutterudites. Note that $1 \mathrm{u}$ does not appear within rank 7 .

The coefficient $T_{m \sigma, m^{\prime} \sigma^{\prime}}^{(k, \gamma)}$ is calculated from the spherical tensor operator as follows. First we change the $f$-electron basis from $(m, \sigma)$ to $(j, \mu)$. Note that $j$ takes $7 / 2$ and $5 / 2$ for $f$ electrons. For a certain value of angular momentum $j$ and its $z$-component $\mu$, the matrix element of spherical tensor operator is easily calculated by the Wigner-Eckart theorem as

$$
\left\langle j \mu\left|T_{q}^{(k)}\right| j \mu^{\prime}\right\rangle=\frac{\left\langle j\left\|T^{(k)}\right\| j\right\rangle}{\sqrt{2 j+1}}\left\langle j \mu \mid j \mu^{\prime} k q\right\rangle,
$$

where $\left\langle J M \mid J^{\prime} M^{\prime} J^{\prime \prime} M^{\prime \prime}\right\rangle$ denotes the Clebsch-Gordan coefficient and $\left\langle j\left\|T^{(k)}\right\| j\right\rangle$ is the reduced matrix element for spherical tensor operator, given by

$$
\left\langle j\left\|T^{(k)}\right\| j\right\rangle=\frac{1}{2^{k}} \sqrt{\frac{(2 j+k+1) !}{(2 j-k) !} .}
$$

Note that $k \leq 2 j$ and the highest rank is $2 j$. The coefficient $T_{m \sigma, m^{\prime} \sigma^{\prime}}^{(k, q)}$ is obtained by returning to the basis of $(m, \sigma)$ from $(j, \mu)$. The final result is given by

$$
\begin{aligned}
T_{m \sigma, m^{\prime} \sigma^{\prime}}^{(k, \gamma)} & =\sum_{j, \mu, \mu^{\prime}, q} G_{\gamma, q}^{(k)} \frac{\left\langle j\left\|T^{(k)}\right\| j\right\rangle}{\sqrt{2 j+1}}\left\langle j \mu \mid j \mu^{\prime} k q\right\rangle \\
& \times\left\langle j \mu \mid \ell m s \frac{\sigma}{2}\right\rangle\left\langle j \mu^{\prime} \mid \ell m^{\prime} s \frac{\sigma^{\prime}}{2}\right\rangle,
\end{aligned}
$$


where $\ell=3, s=1 / 2, j=\ell \pm s$, and $\mu$ runs between $-j$ and $j$.

For the $j$ - $j$ coupling scheme in the $j=7 / 2$ octet, we should discard the contribution from $j=5 / 2$ sextet. Then, the multipole operator in the $j$ - $j$ coupling scheme is expressed in the second-quantized form as

$$
\hat{T}_{\gamma}^{(k)}=\sum_{\mu, \mu^{\prime}} \tilde{T}_{\mu, \mu^{\prime}}^{(k, \gamma)} f_{\mu}^{\dagger} f_{\mu^{\prime}}
$$

where $\mu$ denotes the $z$-component of $j=7 / 2$. The coefficient $\tilde{T}_{\mu, \mu^{\prime}}^{(k, q)}$ is given by

$$
\tilde{T}_{\mu, \mu^{\prime}}^{(k, \gamma)}=\sum_{q} G_{\gamma, q}^{(k)} \frac{\left\langle j\left\|T^{(k)}\right\| j\right\rangle}{\sqrt{2 j+1}}\left\langle j \mu \mid j \mu^{\prime} k q\right\rangle,
$$

where $j$ is fixed as $j=7 / 2$ in this equation. We use this definition for the calculation of the multipole susceptibility in the $j$ - $j$ coupling scheme.

It should be noted here that multipoles belonging to the same symmetry are mixed in general, even if the rank is different. In addition, multipoles are also mixed due to the effect of CEF potentials of the $T_{\mathrm{h}}$ group. Namely, the $f$-electron spin-charge density should be given by the appropriate superposition of multipoles, expressed as

$$
\hat{X}=\sum_{k, \gamma} p_{\gamma}^{(k)} \hat{T}_{\gamma}^{(k)}
$$

In order to determine the coefficient $p_{\gamma}^{(k)}$, it is necessary to evaluate the multipole susceptibility in the linear response theory. However, multipoles belonging to the same symmetry are mixed in general, even if the rank is different. In addition, multipoles are also mixed due to the CEF effect. Thus, it is natural to define $p_{\gamma}^{(k)}$ by the eigenstate of susceptibility matrix

$$
\begin{aligned}
\chi_{k \gamma, k^{\prime} \gamma^{\prime}}= & \frac{1}{Z} \sum_{i, j} \frac{e^{-E_{i} / T}-e^{-E_{j} / T}}{E_{j}-E_{i}}\left\langle i\left|\left[\hat{T}_{\gamma}^{(k)}-\rho_{\gamma}^{(k)}\right]\right| j\right\rangle \\
& \times\left\langle j\left|\left[\hat{T}_{\gamma^{\prime}}^{\left(k^{\prime}\right)}-\rho_{\gamma^{\prime}}^{\left(k^{\prime}\right)}\right]\right| i\right\rangle,
\end{aligned}
$$

where $E_{i}$ is the eigenenergy for the $i$-th eigenstate $|i\rangle$ of $H$ or $\tilde{H}, T$ is a temperature, $\rho_{\gamma}^{(k)}=\sum_{i} e^{-E_{i} / T}\left\langle i\left|\hat{T}_{\gamma}^{(k)}\right| i\right\rangle / Z$, and $Z$ is the partition function given by $Z=\sum_{i} e^{-E_{i} / T}$. Note that the multipole susceptibility is given by the eigenvalue of the susceptibility matrix.

\subsection{Method}

In order to evaluate the multipole susceptibility of the impurity Anderson model, here we employ a numerical renormalization group (NRG) method. ${ }^{49)}$ In this technique, we can include efficiently the conduction electrons states near the Fermi energy by discretizing momentum space logarithmically. Note that in actual calculations, it is necessary to introduce a cut-off $\Lambda$ for the logarithmic discretization of the conduction band. Due to the limitation of computer resources, we keep only $M$ low-energy states. In this paper, we set $\Lambda=6$ and $M=2000$. The temperature $T$ is defined as $T=\Lambda^{-(N-1) / 2}$ in the NRG calculation, where $N$ is the number of the renormalization step. With the use of NRG technique, we evaluate entropy $S_{\mathrm{imp}}$, specific heat $C_{\mathrm{imp}}$, and multipole susceptibility $\chi$. In particular, the optimized multipole state is defined
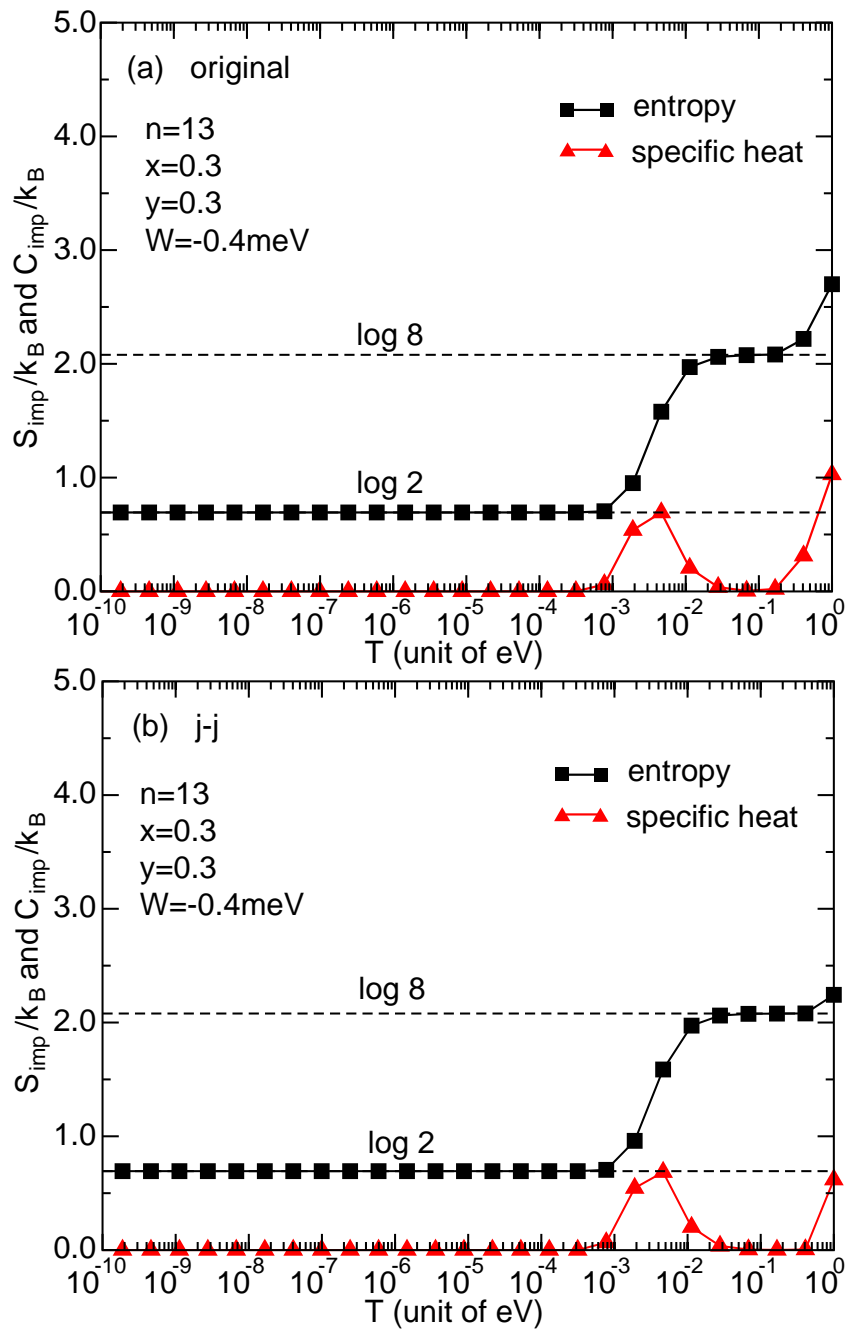

Fig. 3. (Color online) Entropy $S_{\mathrm{imp}}$ and specific heat $C_{\mathrm{imp}}$ of (a) the original seven-orbital model eq. (29) and (b) the $j$ - $j$ coupling model eq. (30) for $n=13$ and $x=0.3$.

by the eigen state with the maximum eigen value of multipole susceptibility matrix eq. (41).

\subsection{Results}

Let us now show our numerical results. First we consider the case of $n=13$. In Figs. 3, we depict the results of entropy and specific heat both for the original seven-orbital Anderson model eq. (29) and the $j$ - $j$ coupling Anderson model eq. (30). Except for the high-temperature region such as $T \sim 1 \mathrm{eV}$, we do not find significant difference between two panels. Since the local $f$-electron number is fluctuating due to the effect of hybridization, the $f$-electron wave function of the original seven-orbital Anderson model is not equal to that of the $j-j$ coupling Anderson model. However, as naively expected from the similarity in the local $f$-electron states, the $j$ - $j$ coupling model can reproduce well the results of the original model.

The difference between both models can be observed at high temperatures as $T \sim 1$ in the region without enough renormalization steps. Since in the $j$ - $j$ coupling model, we discard the $j=5 / 2$ states, it is natural that there appears difference from the original seven-orbital model at high temperatures. Here the energy unit is a half of the conduction bandwidth, which is in the order of eV. Since $\lambda$ is in the order of $0.1 \mathrm{eV}$, it is 

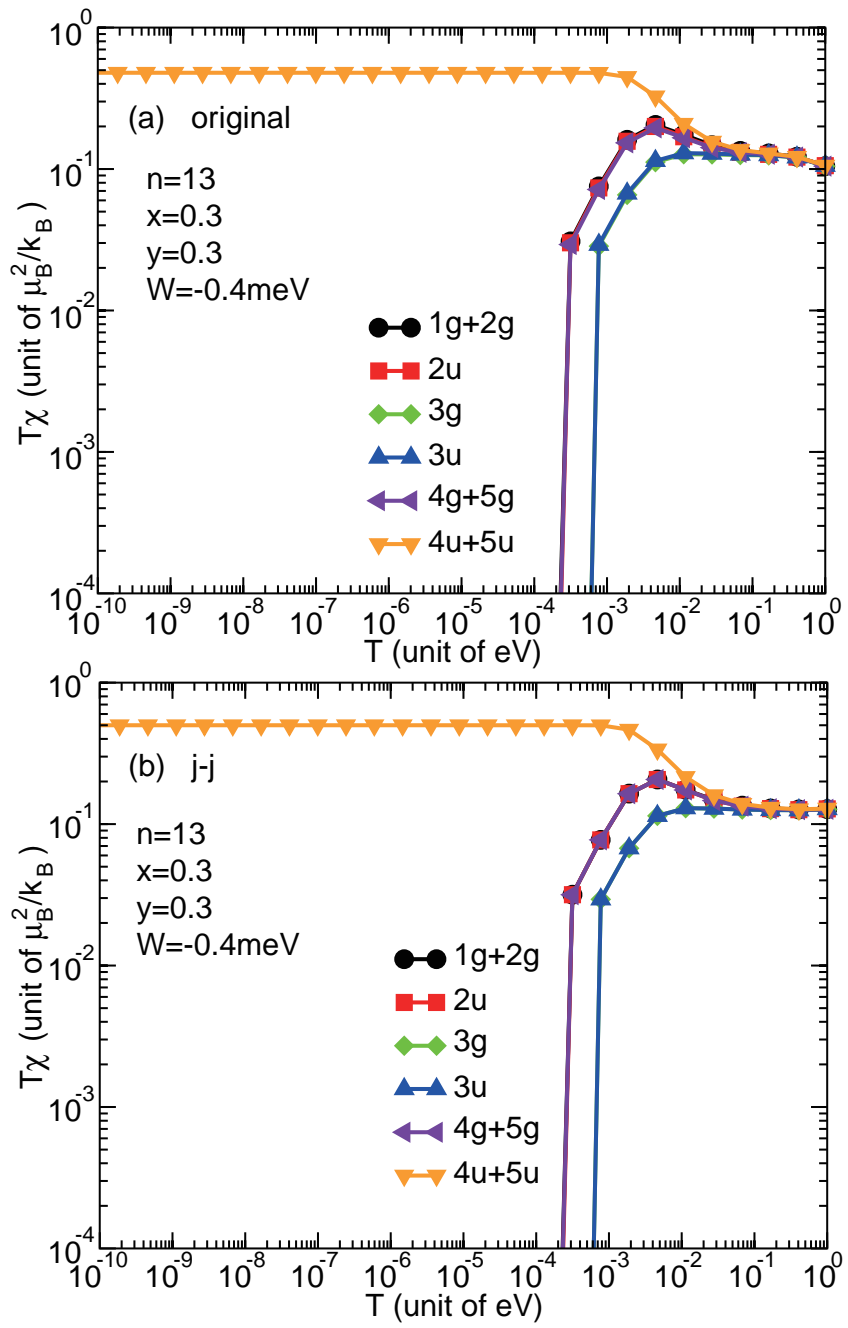

Fig. 4. (Color online) Multipole susceptibilities of (a) the original sevenorbital model eq. (29) and (b) the $j-j$ coupling model eq. (30) for $n=13$ and $x=0.3$.

reasonable that the deviation can be found even at $T>0.1$.

Around at a temperature of 0.1 , we find a plateau in the entropy with the value of $\log 8$. It is easily understood that this is due to 8 -fold degeneracy of $j=7 / 2$, since seven electrons (or one hole) are included in the $j=7 / 2$ octet. A part of the entropy $\log 8$ is released around at $T=0.01 \sim 0.001$ and a peak in the specific heat is found at $T=0.005$. Then, we find residual entropy of $\log 2$ in the temperature region less than $T=0.001$. The appearance of the partial entropy release is understood due to the CEF energy splitting. Namely, as observed in Fig. 1(b), the octet is found to be split in the CEF energy scale in the order of $0.01 \mathrm{eV}$.

In the present Anderson models for filled skutterudites, we consider only the $a_{\mathrm{u}}$ conduction band composed of $p$ electrons in pnictogens. As shown in the explanation of the models, this conduction band is hybridized with $\Gamma_{7}^{-}$state in the $O_{\mathrm{h}}$ group. In the $T_{\mathrm{h}}$ group, $\Gamma_{6}^{-}$and $\Gamma_{7}^{-}$are mixed to form two doublets. Namely, the $\Gamma_{7}^{-}$component of the CEF ground state for $n=13$ in the $T_{\mathrm{h}}$ group is hybridized with the conduction band, while the $\Gamma_{6}^{-}$component remains even at low temperatures. Thus, there occurs residual entropy of $\log 2$ in the present model. Of course, when we consider further the conduction bands other than $a_{\mathrm{u}}$, the residual entropy should

\begin{tabular}{c|c|r|r}
\hline rank $k$ & $\gamma$ & Seven-orbital model & $j$ - $j$ coupling model \\
\hline 1 & $4 \mathrm{u}$ & -0.25091 & -0.29008 \\
\hline 3 & $4 \mathrm{u}$ & 0.18220 & 0.18893 \\
\hline 3 & $5 \mathrm{u}$ & 0.24455 & 0.26034 \\
\hline 5 & $4 \mathrm{u}(1)$ & 0.66679 & 0.65432 \\
\hline 5 & $4 \mathrm{u}(2)$ & 0.11779 & 0.11847 \\
\hline 5 & $5 \mathrm{u}$ & -0.11770 & -0.11974 \\
\hline 7 & $4 \mathrm{u}(1)$ & 0.03571 & 0.03743 \\
\hline 7 & $4 \mathrm{u}(2)$ & -0.57753 & -0.56282 \\
\hline 7 & $5 \mathrm{u}(2)$ & -0.19299 & -0.19418 \\
\hline
\end{tabular}

Table I. Coefficients $p_{\gamma}^{(k)}$ of low-temperature multipole state eq. (40) with the largest eigenvalue in the original seven-orbital model eq. (29) and the $j$ - $j$ coupling model eq. (30) for $n=13$ and $x=0.3$.

be finally released at low temperatures.

Let us move on to the numerical results for multipole susceptibility. In Figs. 4, we show the temperature dependence of eigenvalues $\chi$ of the susceptibility matrix which are classified by the symmetry. Note that we plot $T \chi$, not $\chi$, which is the Curie constant for the multipole susceptibility. In this case, the difference between both models is not so significant even at high temperatures. At low temperatures, the magnitude of multipole susceptibility in Fig. 4(a) is slightly different from that in Fig. 4(b), but its difference is very small.

In order to confirm the effectiveness of the $j-j$ coupling model, let us turn our attention to the multipole states, not the eigenvalues, of the multipole susceptibility. In Table I, we explicitly list the numbers of the component $p_{\gamma}^{(k)}$ of the multipole state at low enough temperatures for both models. First we note that $4 \mathrm{u}$ and $5 \mathrm{u}$ are mixed due to the effect of the $T_{\mathrm{h}}$ group and higher-order multipoles are also included with significant weights. In general, there is no explicit relation between admixture and rank in the multipole state. It is not surprising to obtain significant components of higher-order multipoles. When we compare the value of each component, of course, there exists difference between two models, but we can conclude in a satisfactory level that the multipole state of the original seven-orbital Anderson model is reproduced by the $j-j$ coupling model. Thus, the $j-j$ coupling model is useful to analyze the $f$-electron state with the use of small numbers of relevant $f$ orbitals.

Next we consider the case of $n=12$. First we set $x$ as $x=0.3$ which is considered to be an appropriate value for filled skutterudites $\mathrm{ROs}_{4} \mathrm{Sb}_{12}$, even if rare-earth atom $\mathrm{R}$ is substituted. For $n=12$, as observed in Figs. 2, the CEF ground state is $\Gamma_{1}^{+}$ singlet. In Figs. 5, we show the numerical results of entropy and specific heat both for the original seven-orbital and the $j$ $j$ coupling models. Again we see that both panels agrees well with each other, except for difference in the high-temperature region $T \sim 1$. Thus, we reconfirm that the $j-j$ coupling model works well also for the case of $n=12$ with two $f$ holes.

For a temperature between 0.01 and 0.1 , we observe a short plateau of $\log 13$ in the entropy, which is considered to be due to 13 -fold degeneracy of the $J=6$ state. Then, the entropy of $\log 13$ is released to arrive at the singlet ground state. In the singlet ground state, we expect no multipole moment. In fact, as shown in Figs. 6, multipole susceptibilities vanish at a temperature at which the specific heat shows a peak due to the release of entropy $\log 13$. Note that for the $j-j$ coupling model, at high temperatures, we see significant difference in multi- 

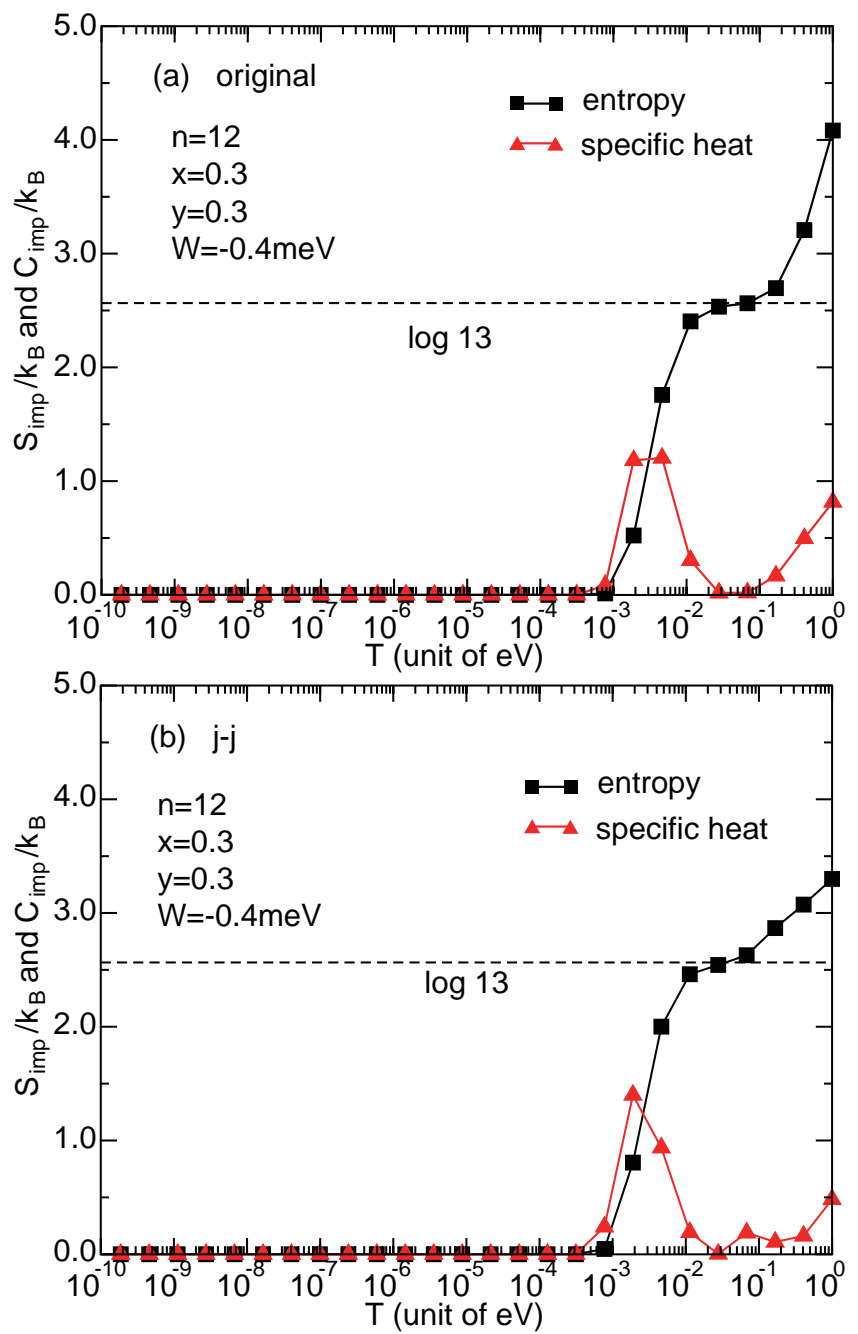

Fig. 5. (Color online) Entropy $S_{\mathrm{imp}}$ and specific heat $C_{\mathrm{imp}}$ of (a) the original model seven-orbital eq. (29) and (b) the $j$ - $j$ coupling model eq. (30) for $n=12$ and $x=0.3$.

pole susceptibilities between Figs. 6(a) and 6(b). Probably it is due to the difference in high-energy local states between the original seven-orbital and the $j$ - $j$ coupling models in the combination with the lack of the renormalization steps. In any case, when we further make the renormalization process, we finally obtain the same behavior in multipole susceptibility.

Even at low enough temperatures, no multipole susceptibility is observed for $n=12$ and $x=0.3$, but it is interesting to consider a possibility of heavy-electron state in Tm-based filled skutterudites. Namely, on the basis of the present numerical calculations, we observe large entropy release such as $\log 13$ at relatively high temperature. It may be risky to conclude the heavy-electron state only from the present results, but it seems to be interesting to perform the measurements of basic bulk properties of Tm-based filled skutterudites, although it may be difficult to synthesize actually Tm-based filled skutterudite compounds.

Let us again turn our attention to multipole state for $n=12$. Here we increase the value of $x$ by assuming that the value of $x$ is controlled experimentally due to the substitution of transition metal atoms and/or pnictogens. In Figs. 7 and 8, we show the numerical results for $n=12$ and $x=1.0$. Note that at $x=1.0$, the local CEF ground state is $\Gamma_{23}^{+}$non-Kramers doublet
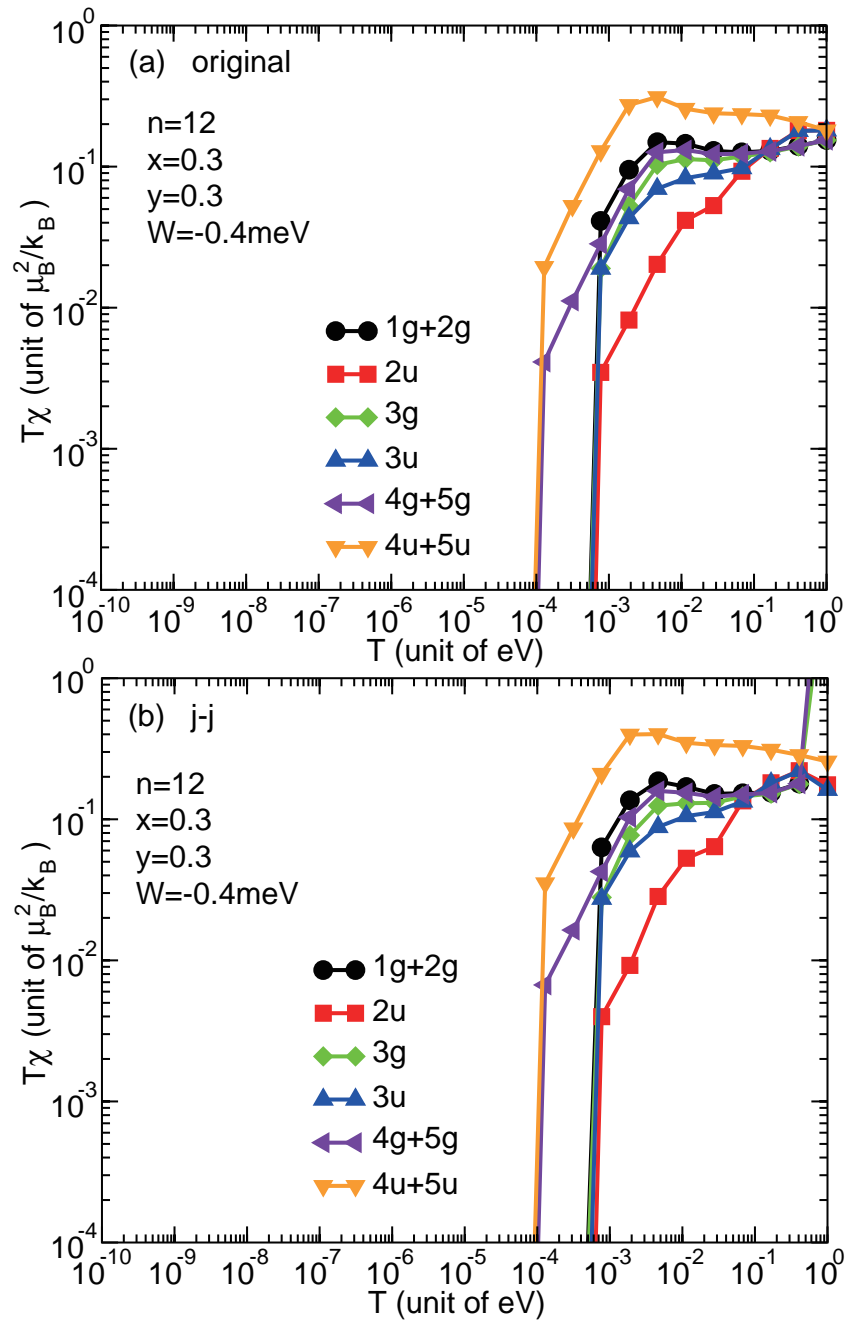

Fig. 6. (Color online) Multipole susceptibilities of (a) the original sevenorbital model eq. (29) and (b) the $j-j$ coupling model eq. (30) for $n=12$ and $x=0.3$.

with the first excited state of $\Gamma_{4}^{+}$triplet, as observed in Figs. 2.

In Fig. 7(a), we show the results of entropy and specific heat. Except for the high-temperature region, we again observe that both panels are similar to each other. In this case, after a short plateau of $\log 13$ around at $T \sim 0.1$, we observe the remnant of plateau of $\log 5$ due to 5 -fold degeneracy of quasi-quintet composed of $\Gamma_{23}^{+}$doublet and $\Gamma_{4}^{+}$triplet. Then, we arrive at the residual doublet state composed of a couple of electrons in $\Gamma_{67}^{-}$quartet state ( $\Gamma_{8}^{-}$in the $O_{\mathrm{h}}$ group). Since in the present model, we consider only the single $a_{\mathrm{u}}$ conduction band, which is hybridized with $\Gamma_{7}^{-}$component of $\Gamma_{5}^{-}$doublet state, $f$ electrons in $\Gamma_{67}^{-}$states are considered to be localized. As mentioned above, in actual materials, the residual $\log 2$ entropy should be finally released, since there exist other conduction bands such as $e_{\mathrm{u}}$ which hybridize with $\Gamma_{67}^{-}$states.

Let us explain the results for multipole susceptibility. It is observed that except for the high-temperature region larger than $T=0.1$, Figs. 8(a) and 8(b) agree well with each other. At high temperatures, significant difference can be found in multipole susceptibilities between Figs. 8(a) and 8(b), but it is due to the same reasons as those in Figs. 6. At low enough temperatures, we find two kinds of residual multipole states which are expected to be dominant in actual materials, although or- 

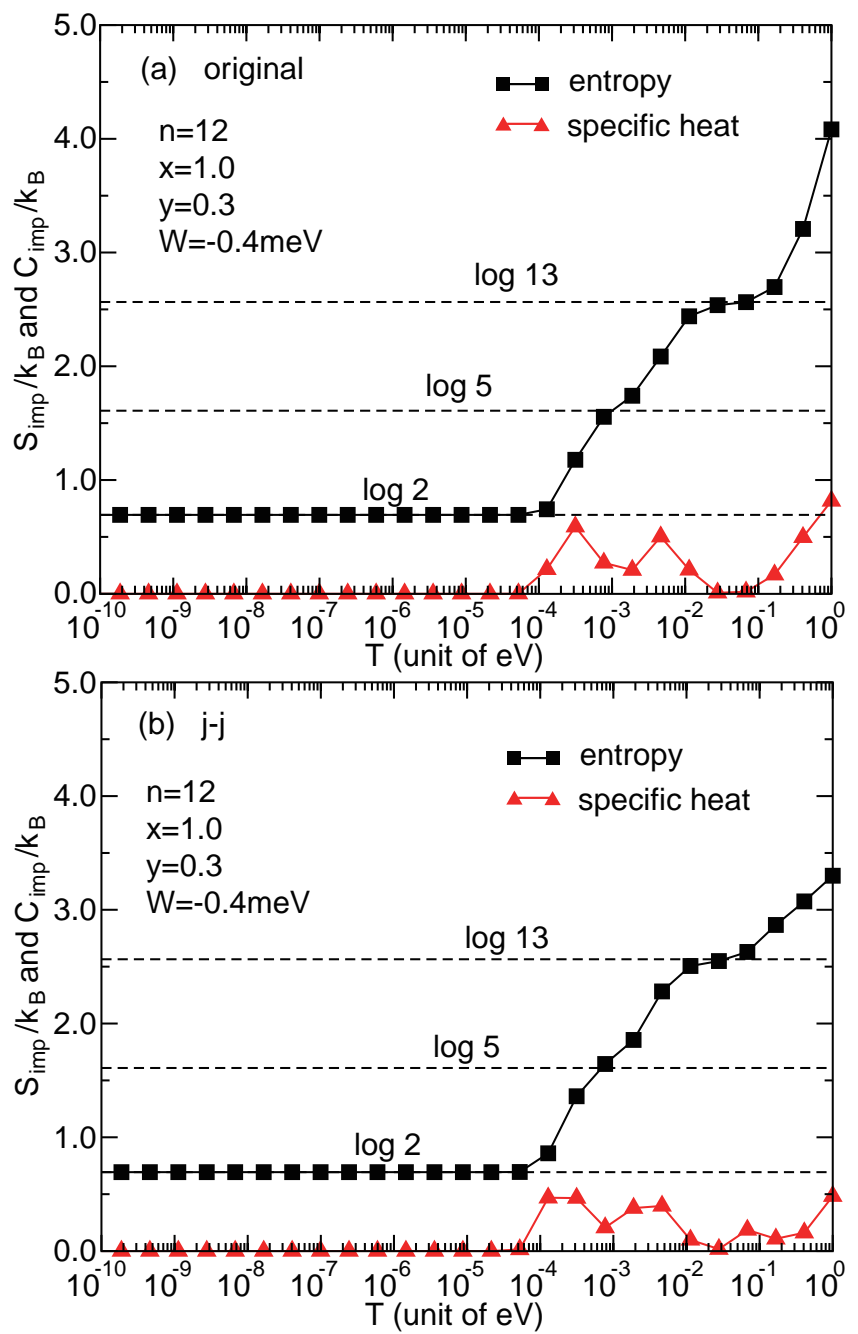

Fig. 7. (Color online) Entropy $S_{\mathrm{imp}}$ and specific heat $C_{\mathrm{imp}}$ of (a) the original model seven-orbital eq. (29) and (b) the $j$ - $j$ coupling model eq. (30) for $n=12$ and $x=1.0$.

\begin{tabular}{c|c|r|r}
\hline rank $k$ & $\gamma$ & Seven-orbital model & $j$ - $j$ coupling model \\
\hline 2 & $3 \mathrm{~g}$ & -0.70572 & -0.69780 \\
\hline 4 & $3 \mathrm{~g}$ & -0.01737 & -0.00369 \\
\hline 6 & $3 \mathrm{~g}$ & 0.70828 & 0.71628 \\
\hline
\end{tabular}

Table II. Coefficients $p_{\gamma}^{(k)}$ of low-temperature multipole state eq. (40) with the largest eigenvalue in the original seven-orbital model eq. (29) and the $j-j$ coupling model eq. (30) for $n=12$ and $x=1.0$.

dering type cannot be specified by the present calculations. The eigenstate with the largest eigenvalue is found to be characterized by $3 \mathrm{~g}$, while the eigenstate with the second largest eigenvalue is labelled by $2 u$.

In order to examine the multipole state, in Table II, we show $p_{\gamma}^{(k)}$ of the eigenstate with the largest eigenvalue. We find small difference in the values between the original sevenorbital and the $j-j$ coupling models, but it can be concluded that the $j-j$ coupling model works well for the description of the multipole state for $n=12$. In the multipole state characterized by $3 \mathrm{~g}$, we find two significant components of rank 2 (quadrupole) and rank 6 (tetrahexacontapole), while the rank 4 component (hexadecapole) is negligibly small. We are in-
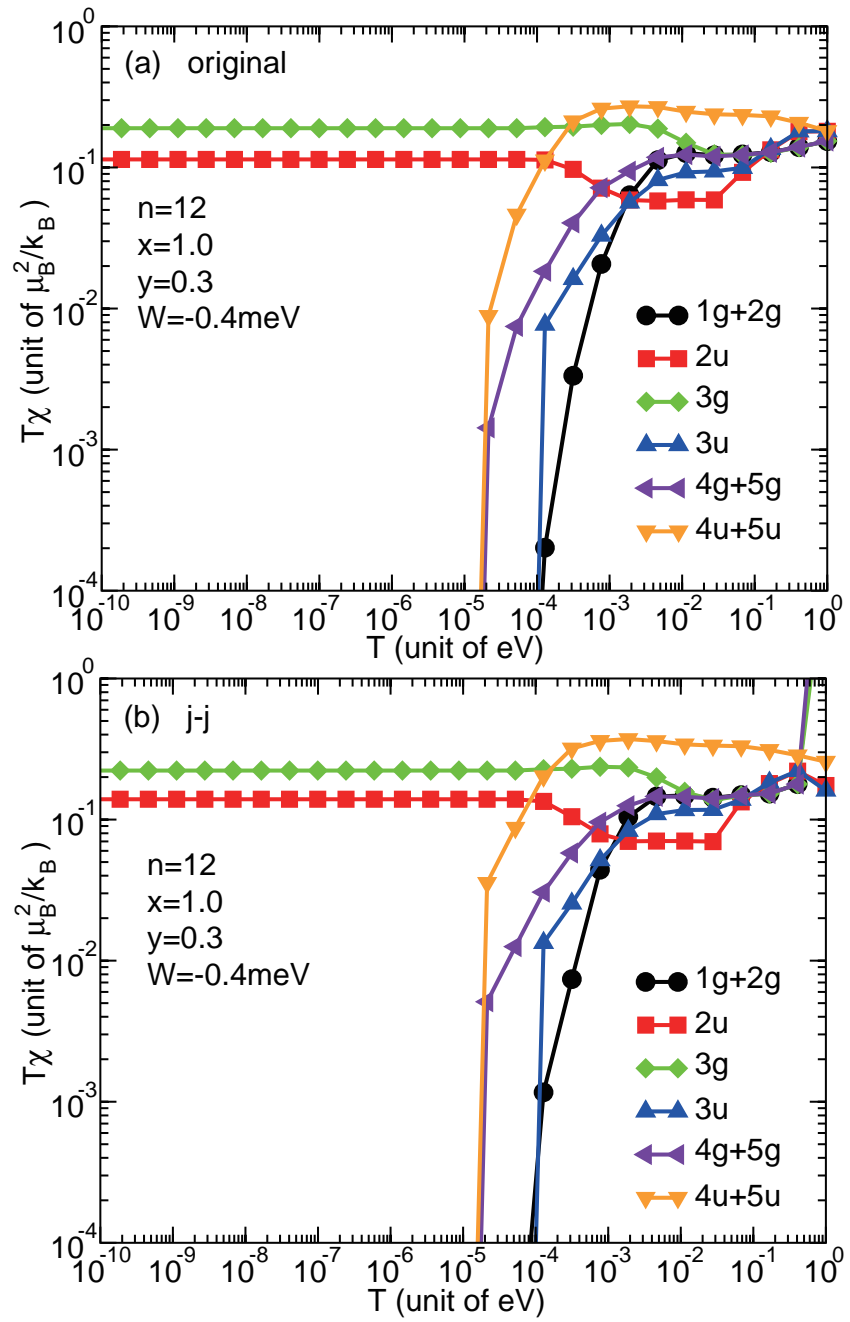

Fig. 8. (Color online) Multipole susceptibilities of (a) the original sevenorbital model eq. (29) and (b) the $j$-j coupling model eq. (30) for $n=12$ and $x=1.0$.

\begin{tabular}{c|c|r|r}
\hline rank $k$ & $\gamma$ & Seven-orbital model & $j$-j coupling model \\
\hline 3 & $2 \mathrm{u}$ & -0.05988 & -0.01126 \\
\hline 7 & $2 \mathrm{u}$ & 0.99821 & 0.99994 \\
\hline
\end{tabular}

Table III. Coefficients $p_{\gamma}^{(k)}$ of low-temperature multipole state eq. (40) with the second largest eigenvalue in the original seven-orbital model eq. (29) and the $j$ - $j$ coupling model eq. (30) for $n=12$ and $x=1.0$.

clined to think that the present $3 \mathrm{~g}$ multipole state is expressed by anti-bonding combination of quadrupole and tetrahexacontapole, although we cannot prove it analytically at this stage.

In Table III, we show the components for the eigenstate with the second largest eigenvalue. This is the multipole characterized by $2 \mathrm{u}$, which is expected to appear in filled skutterudite structure due to the localized nature of electrons in $\Gamma_{67}^{-}{ }^{50,51)}$ Interestingly enough, the main component is not octupole (rank 3) as expected in the case of $n=5,{ }^{50,51)}$ but the rank-7 component (octacosahectapole) becomes dominant. It may be concluded that pure $2 \mathrm{u}$ octacosahectapole occurs from the numerical results of both the original seven-orbital and the $j$ - $j$ coupling models. Such a high-rank multipole has been never observed and a way to detect it experimentally is not 
known. However, we expect that exotic ground state including higher-order multipoles such as rank 6 and 7 is realized in Tm-based filled skutterudites.

\section{Discussion and Summary}

In this paper, we have proposed the microscopic model for $\mathrm{Yb}$ - and Tm-based compounds on the basis of the $j-j$ coupling scheme. We have analyzed the impurity Anderson model in the $j-j$ coupling scheme with the use of a numerical renormalization group technique. The results have indicated that the $j-j$ coupling model works well for the microscopic description of the multi- $f$-electron state.

Note, however, that in the present paper, we have considered only the single conduction band in the Anderson model. Namely, some orbitals are assumed to be localized, but in actual situations, $f$ electrons in all orbitals should be, more or less, hybridized with conduction bands. The present Anderson model is useful to select the candidates of possible multipoles at low temperatures in an unbiased manner, although it is not enough to understand low-temperature properties of actual materials quantitatively.

In order to consider simultaneously the formation of heavy electron state and the appearance of magnetism and/or superconductivity, it is necessary to analyze the periodic Anderson model. It is not difficult to write down the Hamiltonian for the the multiorbital periodic Anderson model on the basis of the present prescription of the $j-j$ coupling scheme for $\mathrm{Yb}$ and Tm compounds. As for magnetism, one way is to derive the orbital dependent RKKY interaction for the determination of the type of multipole ordering. Such calculations may be performed, although it is difficult to discuss the competition with Kondo effect. The actual analysis of the multiorbital periodic Anderson model in the $j-j$ coupling scheme will be an important issue to be resolved in future.

As for emergence of superconductivity, first we assume that heavy-electron states are formed. Then, we consider multiorbital Hubbard-like model for quasi-particles with the local interaction in the $j-j$ coupling scheme, which is written as

$$
\begin{aligned}
H & =\sum_{\boldsymbol{i}, \boldsymbol{a}, \mu, \nu} \tilde{t}_{\mu, \nu}^{\boldsymbol{a}} f_{\boldsymbol{i} \mu}^{\dagger} f_{\boldsymbol{i}+\boldsymbol{a} \nu}+\sum_{\boldsymbol{i}, \mu, \nu} \tilde{B}_{\mu, \nu} f_{\boldsymbol{i} \mu}^{\dagger} f_{\boldsymbol{i} \nu} \\
& +\sum_{\boldsymbol{i}, \mu, \nu, \mu^{\prime}, \nu^{\prime}} \tilde{I}_{\mu, \nu ; \nu^{\prime}, \mu^{\prime}} f_{\boldsymbol{i} \mu}^{\dagger} f_{\boldsymbol{i} \nu}^{\dagger} f_{\boldsymbol{i} \nu^{\prime}} f_{\boldsymbol{i} \mu^{\prime}},
\end{aligned}
$$

where $f_{\boldsymbol{i} \mu}$ is the annihilation operator for $f$ electron at site $i$ with $z$-component $\mu$ of $j=7 / 2, \tilde{t}_{\mu, \nu}^{a}$ is $f$-electron effective hopping between $\mu$ - and $\nu$-orbitals along a direction specified by $\boldsymbol{a}$ connecting adjacent two sites, $\tilde{B}$ is given by eq. (16), and $\tilde{I}$ are given by eqs. (20)-(26). Here $\tilde{t}$ is evaluated by the tight-binding approximation and it is expressed with the use of Slater-Koster integrals, $(f f \sigma),(f f \pi),(f f \delta)$, and $(f f \phi) .{ }^{52,53)}$ The model eq. (42) will be analyzed, for instance, within a random phase approximation. Then, it will be necessary to proceed to the fluctuation-exchange approximation. In any case, we expect the emergence of exotic superconductivity in the vicinity of ordered state. The multipole ordering can be also discussed in the same scheme. It is one of future directions of the research.

We emphasize that microscopic understanding of magnetism and superconductivity is one of important issues in the research field of strongly correlated $f$-electron systems.
The microscopic research will be difficult on the basis of the $L S$ coupling scheme, but it is possible with the use of standard field-theoretical techniques if we exploit the $j-j$ coupling model which has been shown in the present paper. We note that the applicability of the $j-j$ coupling scheme is wider than one has naively expected from the standard textbook and it works even for the realistic parameter region concerning spin-orbit coupling and Coulomb interactions. We expect that the microscopic research on $f$-electron systems can be further pushed in future with the use of the $j-j$ coupling model.

In summary, we have proposed the prescription to construct the effective microscopic model for heavy lanthanide systems such as $\mathrm{Yb}$ and Tm compounds on the basis of the $j-j$ coupling scheme. We have numerically analyzed a couple of Anderson models in which the local interactions at an impurity site are described by using seven $f$ orbitals and the $j-j$ coupling scheme. We have found that entropy, specific heat, and multipole susceptibilities are well reproduced by the $j$ $j$ coupling model. At low enough temperature, the multipole wave function is also well approximated by the $j-j$ coupling scheme in a satisfactorily level for $n=13$ and 12 .

\section{Acknowledgement}

The author thanks Y. Aoki and H. Sato for useful discussions on heavy-fermion compounds. This work has been supported by a Grant-in-Aid for Scientific Research on Innovative Areas "Heavy Electrons" (No. 20102008) of The Ministry of Education, Culture, Sports, Science, and Technology, Japan. The computation in this work has been done using the facilities of the Supercomputer Center of Institute for Solid State Physics, University of Tokyo.

1) Kondo effect and its related phenomena have been reviewed in J. Phys Soc. Jpn. 74 (2005) 1-238.

2) J. Kondo: Prog. Theor. Phys. 32 (1964) 37

3) J. Kondo: Physica 84B (1976) 40.

4) J. Kondo: Physica 84B (1976) 207.

5) M. Ruderman and C. Kittel: Phys. Rev. 96 (1954) 99.

6) T. Kasuya: Prog. Theor. Phys. 16 (1956) 45.

7) K. Yosida: Phys. Rev. 106 (1957) 893.

8) T. Moriya and K. Ueda: Adv. Phys. 49 (2000) 555

9) G. R. Steware: Rev. Mod. Phys. 73 (2001) 797.

10) T. Moriya and K. Ueda: Rep. Prog. Phys. 66 (2003) 1299.

11) H. Q. Yuan, F. M. Grosche, M. Deppe, C. Geibel, G. Sparn and F. Steglich: Science 302 (2003) 2104,

12) H. v. Löhneysen, A. Rösch, M. Vojta and P. Wölfle: Rev. Mod. Phys. 79 (2007) 1015.

13) P. Monthoux, D. Pines and G. G. Lonzarich: Nature (London) 450 (2007) 1177.

14) P. Gengenwart, Q. Si and F. Steglich: Nature Phys. 4 (2008) 186.

15) S. Doniach: Physica 91B (1977) 231

16) F. Steglich, J. Aarts, C. D. Bredl, W. Lieke, D. Meschede, W. Franz and H. Schäfer: Phys. Rev. Lett. 43 (1979) 1892.

17) H. Hegger, C. Petrovic, E. G. Moshopoulou, M. F. Hundley, J. L. Sarrao, Z. Fisk and J. D. Thompson: Phys. Rev. Lett. 84 (2001) 4986.

18) C. Petrovic, R. Movshovich, M. Jaime, P. G. Pagliuso, M. F. Hundley, J. L. Sarrao, Z. Fisk and J. D. Thompson: Europhys. Lett. 53 (2001) 354.

19) C. Petrovic, P. G. Pagliuso, M. F. Hundley, R. Movshovich, J. L. Sarrao, J. D. Thompson, Z. Fisk and P. Monthoux: J. Phys.: Condens. Matter. 13 (2001) L337.

20) S. Nakatsuji, K. Kuga. Y. Machida, T. Tayama, T. Sakakibara, Y. Karaki, H. Ishimoto, S. Yonezawa, Y. Maeno, E. Pearson, G. G. Lonzarich, H. Lee, L. Balicas and Z. Fisk: Nature Phys. 4 (2008) 603.

21) K. Kuga, Y. Karaki, Y. Matsumoto, Y. Machida and S. Nakatsuji: Phys Rev. Lett. 101 (2008) 137004 
22) E. C. T. O'Farrell, D. A. Tompsett, S. E. Sebastian, N. Harrison, C. Capan, L. Balicas, K. Kuga, A. Matsuo, K. Kindo, M. Tokunaga, S. Nakatsuji, G. Csanyi, Z. Fisk and M. L. Sutherland: Phys. Rev. Lett. 102 (2009) 216402.

23) N. Kase, J. Akimitsu, Y. Ishii, T. Suzuki, I. Watanabe, M. Miyazaki, M. Hiraishi, S. Takeshita and R. Kadono: J. Phys. Soc. Jpn. 78 (2009) 073708 .

24) T. Hotta and K. Ueda: Phys. Rev. B 67 (2003) 104518.

25) T. Hotta: Rep. Prog. Phys. 69 (2006) 2061.

26) K. W. H. Stevens: Proc. Phys. Soc. A65 (1952) 209.

27) M. T. Hutchings: Solid State Phys. 16 (1964) 227.

28) K. Takegahara, H. Harima and A. Yanase: J. Phys. Soc. Jpn. 70 (2001) 1190; ibid. 70 (2001) 3468; ibid. 71 (2002) 372.

29) K. R. Lea, M. J. M. Leask and W. P. Wolf: J. Phys. Chem. Solids 23 (1962) 1381.

30) J. C. Slater: Phys. Rev. 34 (1929) 1293.

31) E. U. Condon and G. H. Shortley: Phys. Rev. 37 (1931) 1025.

32) J. A. Gaunt: Phil. Trans. Roy. Soc. A228 (1929) 195.

33) G. Racah: Phys. Rev. 62 (1942) 438.

34) J. C. Slater: Theory of Atomic Structure, (McGraw-Hill, 1960).

35) B. H. Flowers: Proc. R. Soc. Lond. A 212 (1952) 248.

36) A. R. Edmonds and B. H. Flowers: Proc. R. Soc. Lond. A 214 (1952) 515.

37) Note that the definitions of $E_{k}(k=0 \sim 3)$ for the $j=7 / 2$ octet are different from those for the $j=5 / 2$ sextet in Refs. 24 and 25 .

38) A. de-Shalit and I. Talmi: Nuclear Shell Theory, (Academic Press, New York, 1963).
39) Z. Cai, V. Meiser and C. F. Fischer: Phys. Rev. Lett. 68 (1992) 297.

40) E. Eliav, U. Kaldor and Y. Ishikawa: Phys. Rev. A 51 (1995) 225.

41) T. Hotta: J. Phys. Soc. Jpn. 76 (2007) 083705.

42) T. Hotta: Proc. Int. Conf. New Quantum Phenomena in Skutterudite and Related Systems (Skutterudite 2007), J. Phys. Soc. Jpn. 77 (2008) Suppl. A, p. 96.

43) S. Hüfner: Optical Spectra of Transparent Rare Earth Compounds, (Academic Press, New York, 1978).

44) M. Kohgi, K. Iwasa, M. Nakajima, N. Metoki, S. Araki, N. Bernhoeft, J.-M. Mignot, A. Gukasov, H. Sato, Y. Aoki and H. Sugawara: J. Phys. Soc. Jpn. 72 (2003) 1002.

45) K. Kuwahara, K. Iwasa, M. Kohgi, K. Kaneko, S. Araki, N. Metoki, H. Sugawara, Y. Aoki and H. Sato: J. Phys. Soc. Jpn. 73 (2004) 1438.

46) E. A. Goremychkin, R. Osborn, E. D. Bauer, M. B. Maple, N. A. Frederick, W. M. Yuhasz, F. M. Woodward and J. W. Lynn: Phys. Rev. Lett. 93 (2004) 157003.

47) H. Harima and K. Takegahara: J. Phys.: Condens. Matter 15 (2002) S2081.

48) T. Inui, Y. Tanabe and Y. Onodera: Group Theory and Its Applications in Physics, (Springer, Berlin, 1996).

49) H. R. Krishna-murthy, J. W. Wilkins and K. G. Wilson: Phys. Rev. B 21 (1980) 1003.

50) T. Hotta: J. Phys. Soc. Jpn. 76 (2007) 034713.

51) T. Hotta: J. Phys. Soc. Jpn. 77 (2008) 074716.

52) J. C. Slater and G. F. Koster: Phys. Rev. 94 (1954) 1498 (1954).

53) K. Takegahara, Y. Aoki and A. Yanase: J. Phys. C, Solid St. Phys. 13 (1980) 583. 